\title{
Nonperturbative Ball-Chiu construction of the three-gluon vertex
}

\author{
A. C. Aguilar, ${ }^{1}$ M. N. Ferreira, ${ }^{1,2}$ C. T. Figueiredo, ${ }^{1,2}$ and J. Papavassiliou ${ }^{2}$ \\ ${ }^{1}$ University of Campinas-UNICAMP, Institute of Physics "Gleb Wataghin," 13083-859 Campinas, \\ São Paulo, Brazil \\ ${ }^{2}$ Department of Theoretical Physics and IFIC, University of Valencia and CSIC, E-46100 Valencia, Spain
}

(Received 7 March 2019; published 10 May 2019)

\begin{abstract}
We present the detailed derivation of the longitudinal part of the three-gluon vertex from the SlavnovTaylor identities that it satisfies, by means of a nonperturbative implementation of the Ball-Chiu construction; the latter, in its original form, involves the inverse gluon propagator, the ghost dressing function, and certain form factors of the ghost-gluon kernel. The main conceptual subtlety that renders this endeavor nontrivial is the infrared finiteness of the gluon propagator, and the resulting need to separate the vertex into two pieces, one that is intimately connected with the emergence of a gluonic mass scale, and one that satisfies the original set of Slavnov-Taylor identities, but with the inverse gluon propagator replaced by its "kinetic" term. The longitudinal form factors obtained by this construction are presented for arbitrary Euclidean momenta, as well as special kinematic configurations, parametrized by a single momentum. A particularly preeminent feature of the components comprising the tree-level vertex is their considerable suppression for momenta below $1 \mathrm{GeV}$, and the appearance of the characteristic "zero-crossing" in the vicinity of 100-200 MeV. Special combinations of the form factors derived with this method are compared with the results of recent large-volume lattice simulations, and are found to capture faithfully the rather complicated curves formed by the data. A similar comparison with results obtained from Schwinger-Dyson equations reveals a fair overall agreement, but with appreciable differences at intermediate energies. A variety of issues related to the distribution of the pole terms responsible for the gluon mass generation are discussed in detail, and their impact on the structure of the transverse parts is elucidated. In addition, a brief account of several theoretical and phenomenological possibilities involving these newly acquired results is presented.
\end{abstract}

DOI: 10.1103/PhysRevD.99.094010

\section{INTRODUCTION}

The three-gluon vertex of QCD, to be denoted by $\boldsymbol{\Gamma}_{\alpha \mu \nu}$, is inseparably linked with the non-Abelian nature of the theory [1], and is crucial for its most celebrated perturbative property, namely asymptotic freedom [2,3]. In addition, in recent years, the paramount importance of $\boldsymbol{\Gamma}_{\alpha \mu \nu}$ for a plethora of nonperturbative phenomena has become increasingly evident among practitioners, leading to a vigorous activity for unraveling its infrared properties [4-29]. In particular, distinct but equally remarkable aspects of the three-gluon vertex are intimately associated with the emergence of a gluonic mass scale [30-42], the masses and properties of glueballs [43-46], and the potential formation of hybrids and exotics states [47].

Published by the American Physical Society under the terms of the Creative Commons Attribution 4.0 International license. Further distribution of this work must maintain attribution to the author(s) and the published article's title, journal citation, and DOI. Funded by SCOAP .
Perhaps the most intriguing nonperturbative aspect of the three-gluon vertex in the Landau gauge is its so-called "infrared suppression." Specifically, the predominant form factors of $\boldsymbol{\Gamma}_{\alpha \mu \nu}$, which at tree level are equal to unity, decrease gradually as the Euclidean momenta become comparable to the fundamental QCD scale, and eventually reverse their sign, displaying the characteristic "zero-crossing" $[14,15,18,25,28,48]$, finally diverging logarithmically at the origin. These exceptional features have far-reaching theoretical and phenomenological consequences. From the theoretical point of view, the aforementioned behavior of the vertex hinges on the subtle interplay between dynamical effects originating from the two-point sector of the theory $[8,38,49-52]$. In particular, while the gluon acquires dynamically an effective mass, the ghost remains massless even nonperturbatively; thus, loops containing gluons give rise to "protected" logarithms, whilst loops containing ghosts to divergent ones [18]. From the phenomenological perspective, the infrared suppression of $\boldsymbol{\Gamma}_{\alpha \mu \nu}$, and the overall attenuation of the interaction strength that this causes to the Bethe-Salpeter kernels $[43,46]$, appears to be instrumental for the formation of glueball states with masses compatible 
with those obtained from lattice simulations [53]. Moreover, the necessity of a considerable suppression has become evident also in a recent study of the hybrid states, in the framework of the Faddeev equations [47].

At the technical level, the nonperturbative study of the three-gluon vertex is particularly challenging, mainly because it is composed by 14 form factors, which are complicated functions of three independent momenta $(q, r$, and $p)$ [54]. The knowledge of the full momentum dependence of the form factors, in turn, may be crucial for the phenomenological applications mentioned above, essentially because $\boldsymbol{\Gamma}_{\alpha \mu \nu}(q, r, p)$ appears usually inside "loops," and the evaluation of its contribution to the effective strength requires the integration over some of its momenta in the entire range of values. In order to acquire this type of detailed information, one has to turn to continuous approaches, such as the Schwinger-Dyson equations (SDEs) $[10,11,14$ 17,55-59] or the functional renormalization group $[21,22,60]$. Within these latter formalisms, the dynamical equations governing the momentum evolution of the form factors of $\boldsymbol{\Gamma}_{\alpha \mu \nu}$ (or selected subsets thereof) are projected out and solved, usually resorting to certain physically motivated assumptions and judiciously constructed Ansätze, in order to reduce, to some extent, the vast complexity of such undertakings.

In the present work we employ an alternative procedure, which exploits the Slavnov-Taylor identities (STIs), and amounts essentially to a contemporary application of the time-honored method known as "gauge technique" [61-64]. The central idea underlying this approach is to reconstruct the nontransverse part ${ }^{1}$ of the vertex from the quantities that enter in the STIs that $\boldsymbol{\Gamma}_{\alpha \mu \nu}(q, r, p)$ satisfies [see Eq. (2.4)]. In particular, Ball and Chiu (BC) [54] cast the gluon propagator in the form $\Delta^{-1}(q)=q^{2} J_{\mathrm{BC}}(q)$, and express the 10 longitudinal form factors in terms of $J_{\mathrm{BC}}(q)$, the ghost dressing function, $F(q)$, and a subset of the factors comprising the so-called "ghost-gluon scattering kernel", $H_{\nu \mu}$. The nonperturbative structure of all these quantities is in principle known: both $\Delta(q)$ and $F(q)$ have been the focal point of intense investigations in a multitude of studies [33,67-83], while the form factors of $H_{\nu \mu}$ have been computed in a recent work [84], using the one-loop dressed approximation of the SDEs they satisfy.

It turns out, however, that the exercise at hand is considerably more subtle then the simple substitution of the aforementioned ingredients into the $\mathrm{BC}$ solution. The first observation suggesting the need for a nonperturbative "reinterpretation" of the $\mathrm{BC}$ construction stems from the fact that the gluon propagator is infrared finite; then, if the

\footnotetext{
${ }^{1}$ In the original work by Ball and Chiu [54], this part is referred to as "longitudinal", whereas, in some of the more recent literature, the alternative terms "gauge" [65], or "STI saturating" [66] have been put forth as more accurate; throughout this work we adhere to the initial term "longitudinal."
}

$\mathrm{BC}$ parametrization is taken at face value, one realizes immediately that $J_{\mathrm{BC}}(q)$ diverges at the origin as $\Delta^{-1}(0) / q^{2}$. Thus, the "naive" use of the BC solution [54] in the case of an infrared finite gluon propagator would give rise to a longitudinal $\boldsymbol{\Gamma}_{\alpha \mu \nu}$ plagued with poles, which would diverge in the corresponding kinematic limits.

At first sight, this observation alone may not be considered as sufficient cause for readjusting the BC construction; after all, as has been explained in a series of works, the presence of massless poles in $\boldsymbol{\Gamma}_{\alpha \mu \nu}(q, r, p)$ is needed precisely for obtaining an infrared finite solution out of the gluon SDE [33,34,36-41]. The reader must note, however, an important caveat, spelled out in all works cited above: the massless poles contained in $\boldsymbol{\Gamma}_{\alpha \mu \nu}$, comprising a term to be denoted by $V_{\alpha \mu \nu}$, must be of a very special type. In particular, they must be "longitudinally coupled," i.e., appear exclusively in the form $q_{\alpha} / q^{2}, r_{\mu} / r^{2}$, or $p_{\nu} / p^{2}$, or products thereof [see Eq. (2.8)] [36-41]; and this is clearly not the case for the poles induced from the naive use of $J_{\mathrm{BC}}(q)$. In fact, while the former decouple from physical amplitudes and lattice observables, the latter would, in general, persist.

Instead, the self-consistent way to proceed may be briefly described as follows.

(i) One starts by casting the gluon propagator in the form $[36,85]^{2} \quad \Delta^{-1}(q)=q^{2} J(q)+m^{2}(q)$, where $\Delta^{-1}(0)=m^{2}(0) \quad$ (Euclidean space). Evidently, $J_{\mathrm{BC}}(q) \neq J(q)$; in fact, while $J_{\mathrm{BC}}(q)$ diverges as $1 / q^{2}$ at the origin, $J(q)$ diverges only logarithmically, precisely due to the presence of massless ghost loops in its diagrammatic representation.

(ii) The STIs of $\boldsymbol{\Gamma}_{\alpha \mu \nu}(q, r, p)$ [see Eq. (2.4)] will be realized in a very particular way. First, the above form of $\Delta^{-1}(q)$ is substituted on their right-hand side (r.h.s.) Then, on the left-hand side (l.h.s.), $\boldsymbol{\Gamma}_{\alpha \mu \nu}(q, r, p)$ is written as the sum of the pole part, $V_{\alpha \mu \nu}$, and a remainder, denoted by $\Gamma_{\alpha \mu \nu}$. At this point, given that the origin of the terms $m^{2}(q)$ is inextricably connected to the existence of $V_{\alpha \mu \nu}$, it is natural to state that the divergence of $V_{\alpha \mu \nu}$ on the 1.h.s. is responsible for the appearance of the mass terms $m^{2}(q)$ on the r.h.s., while the divergence of $\Gamma_{\alpha \mu \nu}$ accounts for the "kinetic" terms $J(q)$. Thus, each original STI is decomposed into two "partial" ones, one satisfied by $\Gamma_{\alpha \mu \nu}$ and one by $V_{\alpha \mu \nu}$ [see Eqs. (2.10) and (2.11), respectively] [36-41,87].

(iii) This particular decomposition of the STIs converts the original exercise into the following equivalent task. The partial STIs satisfied by $\Gamma_{\alpha \mu \nu}$ are precisely of the type appearing in the original $\mathrm{BC}$ construction

\footnotetext{
${ }^{2}$ Note that, in contradistinction to the more familiar case of the quark propagator, this particular decomposition into a "kinetic" and a "mass" term is not mathematically unique [86].
} 
[54]; indeed, now, on their r.h.s. one has only terms of the type $q^{2} J(q)$, which, up to the aforementioned logarithms, are well behaved in the infrared (have no poles). Thus, the $\mathrm{BC}$ construction may be applied mutatis mutandis for the determination of the longitudinal part of $\Gamma_{\alpha \mu \nu}$.

(iv) As for $V_{\alpha \mu \nu}$, its form is completely determined from the corresponding partial STIs that it satisfies, together with the crucial requirement that it be "longitudinally coupled." Its detailed construction and closed form have been worked out in [37]; see also Eqs. (2.8) and (2.12) of the present article, and the related discussion.

In this work we carry out in the construction described in (i)-(iii), whose careful implementation furnishes the 10 longitudinal form factors of $\Gamma_{\alpha \mu \nu}$, for general values of their Euclidean momenta. The results obtained, in addition to displaying the special features of general infrared suppression, zero crossing, and logarithmic divergence at the origin, compare rather favorably with the lattice data of [25].

The article is organized as follows. In Sec. II we introduce the notation and set up the theoretical framework. We pay particular attention to the connection between $\boldsymbol{\Gamma}_{\alpha \mu \nu}^{a b c}(q, r, p)$ and the mechanism that endows the gluons with a dynamical mass, and introduce the two basic components, $\Gamma_{\alpha \mu \nu}$ and $V_{\alpha \mu \nu}$, together with the "partial" STIs that they satisfy. In Sec. III we present the BC solution for the longitudinal form factors $X_{i}$ of $\Gamma_{\alpha \mu \nu}$, derived from the aforementioned STIs, and comment on the constraints imposed by Bose symmetry. Our main results are presented in Sec. IV, where we explain the theoretical origin of the inputs used in our analysis, present and discuss several threedimensional (3D) and two-dimensional (2D) plots for the $X_{i}$, and compare them with the results of one-loop calculations. Next, in Sec. V we compare our findings with those of previous works based on SDEs, as well as with the two kinematic configurations obtained from recent lattice simulations. In Sec. VI we discuss a series of subtleties related with the construction developed, paying particular attention to the distribution and interpretation of the massless poles. In Sec. VII we elaborate on the complications associated with the "naive" implementation of the BC construction, discussing the necessary adjustments required for its applicability. Sec. VIII is dedicated to a summary of our results, and the discussion of some possible future applications. Finally, in Appendices A and B we present the one-loop results for the form factors in the "totally symmetric" and "asymmetric" configurations, and the transformation rules connecting the $\mathrm{BC}$ and the naive bases.

\section{GENERAL FRAMEWORK AND THEORETICAL FOUNDATIONS}

In this section we introduce the necessary notation and definitions, review certain important relations, and

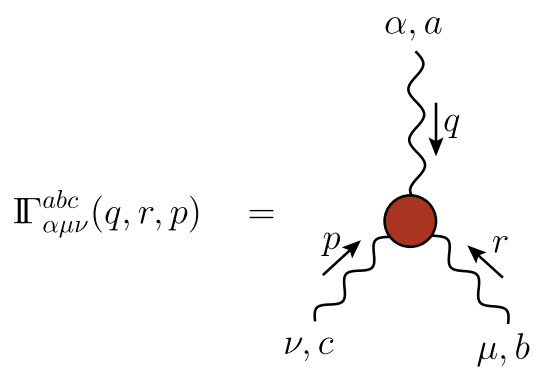

FIG. 1. The full three-gluon vertex with all momenta entering.

elaborate on the main conceptual issues associated with the nonperturbative structure of the three-gluon vertex.

Throughout this article we work in the Landau gauge, where the gluon propagator $\Delta_{\mu \nu}^{a b}(q)=\delta^{a b} \Delta_{\mu \nu}(q)$ assumes the completely transverse form,

$\Delta_{\mu \nu}(q)=-i \Delta(q) P_{\mu \nu}(q), \quad P_{\mu \nu}(q)=g_{\mu \nu}-\frac{q_{\mu} q_{\nu}}{q^{2}}$.

In addition, we introduce the ghost propagator, $D^{a b}(q)=$ $\delta^{a b} D(q)$, whose dressing function, $F(q)$, is given by

$$
D(q)=\frac{i F(q)}{q^{2}} .
$$

The focal point of the present work is the three-gluon vertex, to be denoted by $\boldsymbol{\Gamma}_{\alpha \mu \nu}^{a b c}(q, r, p)=g f^{a b c} \boldsymbol{\Gamma}_{\alpha \mu \nu}(q, r, p)$, which is diagrammatically represented in Fig. 1; note that all momenta are considered to be incoming, so that $q+p+r=0$. At tree level, $\mathbb{I}_{\alpha \mu \nu}(q, r, p):=\Gamma_{\alpha \mu \nu}^{(0)}(q, r, p)$, where

$\Gamma_{\alpha \mu \nu}^{(0)}(q, r, p)=(q-r)_{\nu} g_{\alpha \mu}+(r-p)_{\alpha} g_{\mu \nu}+(p-q)_{\mu} g_{\alpha \nu}$.

The vertex $\boldsymbol{\Gamma}_{\alpha \mu \nu}^{a b c}(q, r, p)$ displays full Bose symmetry, i.e., it remains invariant under the exchange of all "indices" associated with any two of its legs, such as, for example, $(a, \alpha, q) \leftrightarrow(b, \mu, r)$. This fundamental property, in turn, imposes nontrivial constraints on the form factors comprising the three-gluon vertex [see Eqs. (3.7)-(3.9)].

The most important relations for the ensuing analysis are the three STIs that $\boldsymbol{\Gamma}_{\alpha \mu \nu}$ satisfies when contracted by $r^{\mu}, q^{\alpha}$, or $p^{\nu}$, given by [54]

$$
\begin{aligned}
q^{\alpha} \boldsymbol{\Gamma}_{\alpha \mu \nu}(q, r, p)= & F(q)\left[\Delta^{-1}(p) P_{\nu}^{\alpha}(p) H_{\alpha \mu}(p, q, r)\right. \\
& \left.-\Delta^{-1}(r) P_{\mu}^{\alpha}(r) H_{\alpha \nu}(r, q, p)\right], \\
r^{\mu} \boldsymbol{\Gamma}_{\alpha \mu \nu}(q, r, p)= & F(r)\left[\Delta^{-1}(q) P_{\alpha}^{\mu}(q) H_{\mu \nu}(q, r, p)\right. \\
& \left.-\Delta^{-1}(p) P_{\nu}^{\mu}(p) H_{\mu \alpha}(p, r, q)\right], \\
p^{\nu} \boldsymbol{\Gamma}_{\alpha \mu \nu}(q, r, p)= & F(p)\left[\Delta^{-1}(r) P_{\mu}^{\nu}(r) H_{\nu \alpha}(r, p, q)\right. \\
& \left.-\Delta^{-1}(q) P_{\alpha}^{\nu}(q) H_{\nu \mu}(q, p, r)\right] .
\end{aligned}
$$


The $H_{\nu \mu}(q, p, r)$ appearing in the above STIs stands for the ghost-gluon scattering kernel, whose general Lorentz decomposition is given by $[54,88]$

$$
H_{\nu \mu}(q, p, r)=g_{\mu \nu} A_{1}+q_{\mu} q_{\nu} A_{2}+r_{\mu} r_{\nu} A_{3}+q_{\mu} r_{\nu} A_{4}+r_{\mu} q_{\nu} A_{5},
$$

where the momentum dependence of the form factors, $A_{i} \equiv A_{i}(q, p, r)$, has been suppressed for compactness. Notice that, at tree level, $H_{\nu \mu}^{(0)}(q, p, r)=g_{\mu \nu}$, so that $A_{1}^{(0)}=$ 1 and $A_{i}^{(0)}=0$, for $i=2, \ldots, 5$. The nonperturbative structure of the form factors $A_{i}$ is essential for the construction at hand, and has been studied in detail in [84].

Turning now to the relevant dynamical issues, let us first consider the gluon propagator. As has been firmly established through a multitude of studies on the lattice [67-77] and in the continuum [33,78-83], $\Delta(q)$ saturates in the deep infrared at a finite nonvanishing value, i.e., $\Delta^{-1}(0)=c \neq 0$, both in the Landau gauge as well as away from it [89-95]. This characteristic property, in turn, has been interpreted to signal the emergence of a mass scale in the gauge sector of QCD. Motivated by this interpretation, it is natural to cast $\Delta(q)$ in the form (Euclidean space) $[36,85]$

$$
\Delta^{-1}(q)=q^{2} J(q)+m^{2}(q),
$$

where $q^{2} J(q)$ corresponds to the so-called "kinetic term", while $m^{2}(q)$ to an effective (momentum-dependent) gluon mass, with the property $m^{2}(0)=\Delta^{-1}(0)$.

The formalism obtained from the fusion of the pinch technique [4,30,34,96-98] with the background field method (PT-BFM) [99-106] is particularly suited for addressing this fundamental question, by means of the special SDE governing the dynamical evolution of $\Delta(q)$. Within this latter framework, the emergence of a nontrivial $m^{2}(q)$ (i.e., the existence of "massive" solutions) proceeds through a non-Abelian realization of the Schwinger mechanism [107,108], which, in the absence of fundamental scalar fields, endows gauge bosons with masses. The implementation of this mechanism, in turn, hinges crucially on the presence of "longitudinally coupled" massless poles in the vertex $\boldsymbol{\Gamma}_{\alpha \mu \nu}(q, r, p)$, which constitutes a key ingredient of the aforementioned gluon SDE [109-114]. In particular, $\boldsymbol{\Gamma}_{\alpha \mu \nu}(q, r, p)$ is composed by two distinct terms, namely

$$
\boldsymbol{\Gamma}_{\alpha \mu \nu}(q, r, p)=\Gamma_{\alpha \mu \nu}(q, r, p)+V_{\alpha \mu \nu}(q, r, p),
$$

where $V_{\alpha \mu \nu}(q, r, p)$ denotes the part associated with the massless poles, while $\Gamma_{\alpha \mu \nu}(q, r, p)$ captures all remaining contributions. In what follows we briefly summarize some basic properties of $V_{\alpha \mu \nu}(q, r, p)$, which is diagrammatically

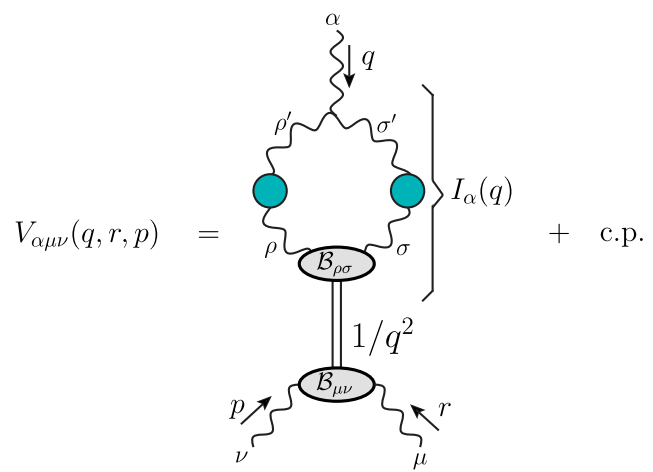

FIG. 2. The pole vertex $V_{\alpha \mu \nu}(q, r, p)$ is composed of three main ingredients: the transition amplitude, $I_{\alpha}(q)$, which mixes the gluon with a massless excitation, the propagator of the massless excitation $1 / q^{2}$, while $\mathcal{B}_{\mu \nu}$ (and $\mathcal{B}_{\rho \sigma}$ ) denotes the proper vertex that couples the massless excitation to a pair of gluons, and "c.p." stands for "cyclic permutations".

represented in the Fig. 2; for further details, the reader is referred to the related literature $[36,37,40,41,87]$.

(i) The origin of the massless poles is dynamical rather than kinematic, in the sense that, for sufficiently strong binding, the mass of certain colored bound states may be reduced to zero [109-114]. The actual nonperturbative realization of this possibility within a contemporary QCD framework has been demonstrated in [36-41,87], where the homogeneous Bethe-Salpeter equation (BSE) that controls the formation of these bound states was investigated.

(ii) The term "longitudinally coupled" means that $V_{\alpha \mu \nu}(q, r, p)$ assumes the very special form

$$
\begin{aligned}
V_{\alpha \mu \nu}(q, r, p)= & \left(\frac{q_{\alpha}}{q^{2}}\right) A_{\mu \nu}(q, r, p)+\left(\frac{r_{\mu}}{r^{2}}\right) B_{\alpha \nu}(q, r, p) \\
& +\left(\frac{p_{\nu}}{p^{2}}\right) C_{\alpha \mu}(q, r, p),
\end{aligned}
$$

and therefore, $V_{\alpha \mu \nu}(q, r, p)$ satisfies the crucial relation

$$
P_{\alpha \alpha^{\prime}}(q) P_{\mu \mu^{\prime}}(r) P_{\nu \nu^{\prime}}(p) V^{\alpha \mu \nu}(q, r, p)=0 .
$$

(iii) We emphasize that the form of $V_{\alpha \mu \nu}(q, r, p)$ given in Eq. (2.8) emerges automatically in the dynamical framework put forth in the classic works of [109-114], and its contemporary variations, studied in [36-41]. In particular, the pole in the $q$-channel is due to the propagation of a massless bound state excitation [87], as shown in the diagram of the Fig. 2; the poles in the other two channels are obtained through the cyclic permutations imposed by the Bose-symmetry of $V_{\alpha \mu \nu}(q, r, p)$. We emphasize that, within this scenario, Lorentz invariance alone forces the saturation of $q$ by its own Lorentz index $\alpha$; in other 
words, the special form of Eq. (2.8) is not assumed, but, instead, emerges automatically.

(iv) Note, in addition, that the pivotal property of Eq. (2.9) guarantees the decoupling of the massless excitations from physical "on-shell" amplitudes, as well as its vanishing from the transversely projected version of $\boldsymbol{\Gamma}_{\alpha \mu \nu}(q, r, p)$, which constitutes the natural ingredient of lattice observables, such as those considered in Sec. V B [see Eqs. (5.1) and (5.2)].

(v) A crucial one-to-one correspondence between $m^{2}(q)$ and $V_{\alpha \mu \nu}(q, r, p)$ is imposed by the need to evade, in a STI preserving way, the so-called "seagull-identity" $[38,39,115]$. Specifically, if one substitutes Eq. (2.7) into the 1.h.s and $\Delta^{-1}(q)=q^{2} J(q)-m^{2}(q)$ (Minkowski space) into the r.h.s. of Eq. (2.4), the full STI must be realized as the sum of two specific pieces, namely

$$
\begin{aligned}
q^{\alpha} \Gamma_{\alpha \mu \nu}(q, r, p)= & F(q)\left[p^{2} J(p) P_{\nu}^{\alpha}(p) H_{\alpha \mu}(p, q, r)\right. \\
& \left.-r^{2} J(r) P_{\mu}^{\alpha}(r) H_{\alpha \nu}(r, q, p)\right],
\end{aligned}
$$

and

$$
\begin{aligned}
q^{\alpha} V_{\alpha \mu \nu}(q, r, p)= & F(q)\left[m^{2}(r) P_{\mu}^{\alpha}(r) H_{\alpha \nu}(r, q, p)\right. \\
& \left.-m^{2}(p) P_{\nu}^{\alpha}(p) H_{\alpha \mu}(p, q, r)\right] .
\end{aligned}
$$

Evidently, two additional pairs of similar (cyclically permuted) relations are obtained from the other two STIs of Eq. (2.4).

(vi) It turns out that Eq. (2.9), together with Eq. (2.11) and the other two cyclic relations, determine completely the form of $V_{\alpha \mu \nu}(q, r, p)$ [37]; in particular,

$$
\begin{aligned}
& A_{\mu \nu}(q, r, p)=\frac{F\left(q^{2}\right)}{2}\left\{m^{2}\left(r^{2}\right) P_{\mu}^{\rho}(r)\left[g_{\nu}^{\sigma}+P_{\nu}^{\sigma}(p)\right] H_{\rho \sigma}(r, q, p)-m^{2}\left(p^{2}\right) P_{\nu}^{\rho}(p)\left[g_{\mu}^{\sigma}+P_{\mu}^{\sigma}(r)\right] H_{\rho \sigma}(p, q, r)\right\}, \\
& B_{\alpha \nu}(q, r, p)=\frac{F\left(r^{2}\right)}{2}\left\{m^{2}\left(p^{2}\right) P_{\nu}^{\rho}(p)\left[g_{\alpha}^{\sigma}+P_{\alpha}^{\sigma}(q)\right] H_{\rho \sigma}(p, r, q)-m^{2}\left(q^{2}\right) P_{\alpha}^{\rho}(q)\left[g_{\nu}^{\sigma}+P_{\nu}^{\sigma}(p)\right] H_{\rho \sigma}(q, r, p)\right\}, \\
& C_{\alpha \mu}(q, r, p)=\frac{F\left(p^{2}\right)}{2}\left\{m^{2}\left(q^{2}\right) P_{\alpha}^{\rho}(q)\left[g_{\mu}^{\sigma}+P_{\mu}^{\sigma}(r)\right] H_{\rho \sigma}(q, p, r)-m^{2}\left(r^{2}\right) P_{\mu}^{\rho}(r)\left[g_{\alpha}^{\sigma}+P_{\alpha}^{\sigma}(q)\right] H_{\rho \sigma}(r, p, q)\right\} .
\end{aligned}
$$

Clearly, the substitution of the above terms into Eq. (2.8) gives rise to a $V_{\alpha \mu \nu}(q, r, p)$ that is manifestly Bose-symmetric. Note that $V_{\alpha \mu \nu}(q, r, p)$ contains single-, double-, and triple-pole terms, such as, for example, $\frac{q_{\alpha} g_{\mu \nu}}{q^{2}}, \frac{q_{\alpha} r_{\mu} r_{\nu}}{q^{2} r^{2}}$, and $\frac{q_{\alpha} r_{\mu} p_{\nu}}{q^{2} r^{2} p^{2}}$ [37]. On the other hand, terms having their momenta and Lorentz indices "mismatched," e.g., $\frac{p_{\alpha} g_{\mu \nu}}{q^{2}}$ or $\frac{r_{\alpha} p_{\mu} q_{\nu}}{q^{2} r^{2} p^{2}}$, are absent.

After this brief review, we return to the central objective of this work, namely the implementation of the BC solution in order to reconstruct the longitudinal part of $\Gamma_{\alpha \mu \nu}(q, r, p)$ from Eq. (2.10) and the other two similar STIs obtained from the contraction by $r^{\mu}$ and $p^{\nu}$. However, before embarking into the technical details of this construction, it is important to clarify an essential point regarding the central ingredient of the $\mathrm{BC}$ solution for $\Gamma_{\alpha \mu \nu}(q, r, p)$, namely the function $J(q)$, and, in particular, the way in which it may be actually computed.

To that end, note that the special decomposition of the $\boldsymbol{\Gamma}_{\alpha \mu \nu}(q, r, p)$ given in Eq. (2.7) leads to the separation of the SDE for $\Delta(q)$ into two individual but coupled integral equations, governing the evolution of $J(q)$ and $m^{2}(q)$ $[36,86]$. It turns out that the components of the equation that determines $m^{2}(q)$ are considerably better known than those entering in the equation for $J(q)$; in particular, the four-gluon vertex drops practically out from the former, but is present in the latter. Therefore, given these practical limitations, one proceeds as follows. First, the equation for $m^{2}(q)$ is solved in isolation, using as input the lattice data for $\Delta(q)$, together with certain simplifying assumptions related to multiplicative renormalization. Then, one employs Eq. (2.6) once again, and obtains $q^{2} J(q)$ by subtracting the solution for $m^{2}(q)$ from the lattice data for $\Delta^{-1}(q)$ [70]. A different, but theoretically equivalent procedure, involves the derivation of a special BSE, whose solution is identified with the first derivative of $\mathrm{m}^{2}(q)$ [40,87]; then, numerical integration furnishes $m^{2}(q)$, and its subtraction from $\Delta^{-1}(q)$, exactly as before, furnishes $q^{2} J(q)$. In Sec. IV we will further elaborate on the structure of $J(q)$, its characteristic properties, and the uncertainties in its determination.

\section{THE BALL-CHIU SOLUTION FOR $\boldsymbol{\Gamma}_{\alpha \mu \nu}(q, \boldsymbol{r}, \boldsymbol{p})$}

For the actual construction of the vertex $\Gamma_{\alpha \mu \nu}(q, r, p)$, let us cast it in the form

$$
\Gamma^{\alpha \mu \nu}(q, r, p)=\Gamma_{\mathbf{L}}^{\alpha \mu \nu}(q, r, p)+\Gamma_{\mathbf{T}}^{\alpha \mu \nu}(q, r, p),
$$

where the "longitudinal" part, $\Gamma_{\mathbf{L}}^{\alpha \mu \nu}(q, r, p)$, saturates the relevant STIs [Eq. (2.10) and c.p. thereof], while the totally "transverse" part, $\Gamma_{\mathbf{T}}^{\alpha \mu \nu}(q, r, p)$, satisfies 
$q_{\alpha} \Gamma_{\mathbf{T}}^{\alpha \mu \nu}(q, r, p)=r_{\mu} \Gamma_{\mathbf{T}}^{\alpha \mu \nu}(q, r, p)=p_{\nu} \Gamma_{\mathbf{T}}^{\alpha \mu \nu}(q, r, p)=0$.

For the explicit tensorial decomposition of $\Gamma_{\mathbf{L}}^{\alpha \mu \nu}(q, r, p)$ and $\Gamma_{\mathbf{T}}^{\alpha \mu \nu}(q, r, p)$ we will employ the Bose symmetric basis introduced in [54]. Specifically,
$\Gamma_{\mathbf{L}}^{\alpha \mu \nu}(q, r, p)=\sum_{i=1}^{10} X_{i}(q, r, p) \ell_{i}^{\alpha \mu \nu}$

where the tensors $\ell_{i}^{\alpha \mu \nu}$ are given by

$\begin{array}{llll}\ell_{1}^{\alpha \mu \nu}=(q-r)^{\nu} g^{\alpha \mu}, & \ell_{2}^{\alpha \mu \nu}=-p^{\nu} g^{\alpha \mu}, & & \ell_{3}^{\alpha \mu \nu}=(q-r)^{\nu}\left[q^{\mu} r^{\alpha}-(q \cdot r) g^{\alpha \mu}\right], \\ \ell_{4}^{\alpha \mu \nu}=(r-p)^{\alpha} g^{\mu \nu}, & \ell_{5}^{\alpha \mu \nu}=-q^{\alpha} g^{\mu \nu}, & \ell_{6}^{\alpha \mu \nu}=(r-p)^{\alpha}\left[r^{\nu} p^{\mu}-(r \cdot p) g^{\mu \nu}\right], & \\ \ell_{7}^{\alpha \mu \nu}=(p-q)^{\mu} g^{\alpha \nu}, & \ell_{8}^{\alpha \mu \nu}=-r^{\mu} g^{\alpha \nu}, & \ell_{9}^{\alpha \mu \nu}=(p-q)^{\mu}\left[p^{\alpha} q^{\nu}-(p \cdot q) g^{\alpha \nu}\right], & \ell_{10}^{\alpha \mu \nu}=q^{\nu} r^{\alpha} p^{\mu}+q^{\mu} r^{\nu} p^{\alpha},\end{array}$

and

$$
\Gamma_{\mathbf{T}}^{\alpha \mu \nu}(q, r, p)=\sum_{i=1}^{4} Y_{i}(q, r, p) t_{i}^{\alpha \mu \nu}
$$

with the $t_{i}^{\alpha \mu \nu}$ given by

$$
\begin{aligned}
t_{1}^{\alpha \mu \nu} & =\left[(q \cdot r) g^{\alpha \mu}-q^{\mu} r^{\alpha}\right]\left[(r \cdot p) q^{\nu}-(q \cdot p) r^{\nu}\right], \\
t_{2}^{\alpha \mu \nu} & =\left[(r \cdot p) g^{\mu \nu}-r^{\nu} p^{\mu}\right]\left[(p \cdot q) r^{\alpha}-(r \cdot q) p^{\alpha}\right], \\
t_{3}^{\alpha \mu \nu} & =\left[(p \cdot q) g^{\nu \alpha}-p^{\alpha} q^{\nu}\right]\left[(q \cdot r) p^{\mu}-(p \cdot r) q^{\mu}\right], \\
t_{4}^{\alpha \mu \nu} & =g^{\mu \nu}\left[(r \cdot q) p^{\alpha}-(p \cdot q) r^{\alpha}\right]+g^{\nu \alpha}\left[(p \cdot r) q^{\mu}-(q \cdot r) p^{\mu}\right]+g^{\alpha \mu}\left[(q \cdot p) r^{\nu}-(r \cdot p) q^{\nu}\right]+r^{\alpha} p^{\mu} q^{\nu}-p^{\alpha} q^{\mu} r^{\nu} .
\end{aligned}
$$

At tree level, the only nonvanishing form factors are

$$
X_{1}^{(0)}(q, r, p)=X_{4}^{(0)}(q, r, p)=X_{7}^{(0)}(q, r, p)=1 .
$$

Bose symmetry with respect to the three legs requires that $\Gamma_{\mathbf{L}}$ reverses sign under the interchange of the corresponding Lorentz indices and momenta (remember that the color factor $f^{a b c}$ has been factored out); this, in turn, imposes the following relations under the exchange of arguments [54]

$$
\begin{array}{cccc}
X_{1}(q, r, p)=X_{1}(r, q, p), & X_{2}(q, r, p)=-X_{2}(r, q, p), & X_{3}(q, r, p)=X_{3}(r, q, p), \\
X_{4}(q, r, p)=X_{4}(q, p, r), & X_{5}(q, r, p)=-X_{5}(q, p, r), & X_{6}(q, r, p)=X_{6}(q, p, r), \\
X_{7}(q, r, p)=X_{7}(p, r, q), & X_{8}(q, r, p)=-X_{8}(p, r, q), & X_{9}(q, r, p)=X_{9}(p, r, q), \\
X_{10}(q, r, p)=-X_{10}(r, q, p), & X_{10}(q, r, p)=-X_{10}(q, p, r), & X_{10}(q, r, p)=-X_{10}(p, r, q) .
\end{array}
$$

In addition, Bose symmetry furnishes the following relations between different form factors [54]

$$
\begin{array}{lll}
X_{4}(q, r, p)=X_{1}(r, p, q), & X_{5}(q, r, p)=X_{2}(r, p, q), & X_{6}(q, r, p)=X_{3}(r, p, q), \\
X_{7}(q, r, p)=X_{1}(p, q, r), & X_{8}(q, r, p)=X_{2}(p, q, r), & X_{9}(q, r, p)=X_{3}(p, q, r),
\end{array}
$$

which reduce the number of independent form factors from the original ten to only four, namely $X_{1}, X_{2}, X_{3}$, and $X_{10}$. In particular, if the dependence of $X_{1}, X_{2}, X_{3}$ on $(q, r, p)$ could be cast in a closed functional form, then all other $X_{i}$ would be obtained from them through a simple interchange of the appropriate momenta, according to Eq. (3.8). However, in practice, $X_{1}, X_{2}, X_{3}$, and $X_{10}$ are computed numerically, and the reconstruction of the remaining $X_{i}$ requires a modest amount of additional numerical effort; a concrete example of how to obtain $X_{4}$ from $X_{1}$ will be given in Sec. IV C. 
For the transverse part, Bose symmetry implies that [54]

$$
\begin{aligned}
& Y_{1}(q, r, p)=Y_{1}(r, q, p), \quad Y_{2}(q, r, p)=Y_{2}(q, p, r), \\
& Y_{3}(q, r, p)=Y_{3}(p, r, q),
\end{aligned}
$$

and

$$
Y_{2}(q, r, p)=Y_{1}(r, p, q), \quad Y_{3}(q, r, p)=Y_{1}(p, q, r) .
$$

Therefore, there are only two independent transverse form factors, $Y_{1}$ and $Y_{4}$.

The form factors $X_{i}$ are fully determined in terms of the $A_{j}, F(q)$, and $J(q)$ by solving the system of linear equations generated by the identity given in Eq. (2.10) and its cyclic permutations. Specifically, the solutions for $X_{1}$, $X_{2}, X_{3}$, and $X_{10}$, first obtained in Ref. [54], read

$$
\begin{aligned}
X_{1}(q, r, p)= & \frac{1}{4}\left[2\left(a_{p q r}+a_{p r q}\right)+p^{2}\left(b_{q r p}+b_{r q p}\right)\right. \\
& +2\left(q \cdot p d_{p r q}+r \cdot p d_{p q r}\right) \\
& \left.+\left(q^{2}-r^{2}\right)\left(b_{r p q}+b_{p q r}-b_{q p r}-b_{p r q}\right)\right], \\
X_{2}(q, r, p)= & \frac{1}{4}\left[2\left(a_{p r q}-a_{p q r}\right)-\left(q^{2}-r^{2}\right)\left(b_{q r p}+b_{r q p}\right)\right. \\
& +2\left(q \cdot p d_{p r q}-r \cdot p d_{p q r}\right) \\
& \left.+p^{2}\left(b_{p r q}-b_{p q r}+b_{q p r}-b_{r p q}\right)\right], \\
X_{3}(q, r, p)= & \frac{1}{q^{2}-r^{2}}\left[a_{r p q}-a_{q p r}+r \cdot p d_{q p r}-q \cdot p d_{r p q}\right], \\
X_{10}(q, r, p)= & -\frac{1}{2}\left[b_{q r p}+b_{r p q}+b_{p q r}-b_{q p r}-b_{r q p}-b_{p r q}\right],
\end{aligned}
$$

where we introduce the following compact notation

$$
\begin{aligned}
a_{\text {qrp }} & \equiv F(r) J(p) A_{1}(p, r, q), \\
b_{q r p} & \equiv F(r) J(p) A_{3}(p, r, q), \\
d_{\text {qrp }} & \equiv F(r) J(p)\left[A_{4}(p, r, q)-A_{3}(p, r, q)\right] .
\end{aligned}
$$

Clearly, these expressions satisfy the exchange symmetries of Eq. (3.7). The remaining six $X_{i}$ may be computed by permuting the arguments, according to Eq. (3.8).

Notice that if the contributions from the ghost sector are turned off, by setting $H_{\nu \mu}(q, p, r)=g_{\nu \mu}$ and $F(q)=1$ into Eqs. (3.11), we obtain the "Abelianized" form factors, $\hat{X}_{i}(q, r, p)$ (in Minkowski space),

$$
\begin{array}{ll}
\hat{X}_{1}(q, r, p)=\frac{1}{2}[J(r)+J(q)], & \hat{X}_{3}(q, r, p)=\frac{[J(q)-J(r)]}{q^{2}-r^{2}}, \\
\hat{X}_{2}(q, r, p)=\frac{1}{2}[J(q)-J(r)], & \hat{X}_{10}(q, r, p)=0 .
\end{array}
$$

Evidently, the above expressions display the correct Bose symmetry properties required by Eqs. (3.7) and (3.8). In fact, this is exactly the result of the $\mathrm{BC}$ construction for a vertex with three "background" gluons, which satisfies "Abelian" Ward identities [4,6,9,12]; of course, in that case, the additional replacement $J(q) \rightarrow J(q)[1+G(q)]^{-2}$ must be carried out, where the function $1+G(q)$ has been studied extensively in the literature [33,34,52,116,117].

Let us finally emphasize that, as long as the quantities appearing on the r.h.s. of Eq. (3.11) are properly renormalized, the resulting $X_{i}(q, r, p)$ will be free of ultraviolet divergences. This is indeed the case, given that $F(q)$, $J(q)$, and the form factors $A_{i}(q, p, r)$ have been duly renormalized [84], using a particular version of the general momentum subtraction (MOM) scheme, known as "Taylor scheme" [118].

In particular, let the renormalization constants of the gluon and ghost propagators and the ghost-gluon and threegluon vertices be defined as [17]

$$
\begin{aligned}
\Delta_{R}(q) & =Z_{A}^{-1} \Delta(q), \\
F_{R}(q) & =Z_{c}^{-1} F(q), \\
\Gamma_{R}^{\mu}(q, p, r) & =Z_{1} \Gamma^{\mu}(q, p, r), \\
\Gamma_{R}^{\alpha \mu \nu}(q, r, p) & =Z_{3} \Gamma^{\alpha \mu \nu}(q, r, p),
\end{aligned}
$$

where the subscript " $R$ " denotes renormalized quantities.

In the Taylor scheme, the renormalization constants $Z_{A}$ and $Z_{c}$ are defined by imposing tree-level values for the propagators at $\mu$, i.e., $F(\mu)=1$ and $J(\mu)=1{ }^{3}$ while $A_{1}$ assumes its tree-level value in the "soft-ghost" kinematics, where Taylor's theorem is valid (Landau gauge), and therefore one sets $Z_{1}=1$ [119]. Then, the remaining renormalization constant, $Z_{3}$, is completely determined by appealing to the STI of Eq. (2.4), which implies that

$$
Z_{3}=Z_{A} Z_{1} Z_{c}^{-1}
$$

As a consequence of this particular choice, the results for the three-gluon vertex will not match exactly those obtained by renormalizing $X_{1}$ in the "totally symmetric" configuration, $q^{2}=p^{2}=r^{2}=\mu^{2}$, often used in the literature. Note, however, that, as has been explicitly shown in [84], when $X_{1}$ is renormalized in the Taylor scheme and subsequently evaluated at the symmetric point, it departs from unity only by about $3 \%$ (for $\mu=4.3 \mathrm{GeV}$ ).

\footnotetext{
${ }^{3}$ Strictly speaking, given the form of $\Delta^{-1}(q)$ in Eq. (2.6), at $\mu$ one must impose the condition $\Delta^{-1}(\mu)=\mu^{2}+m^{2}(\mu)$, which yields $J(\mu)=1$; however, in practice, the same result emerges by imposing simply $\Delta^{-1}(\mu)=\mu^{2}$, given that, at $\mu=4.3 \mathrm{GeV}$, $m^{2}(\mu)$ is negligible.
} 

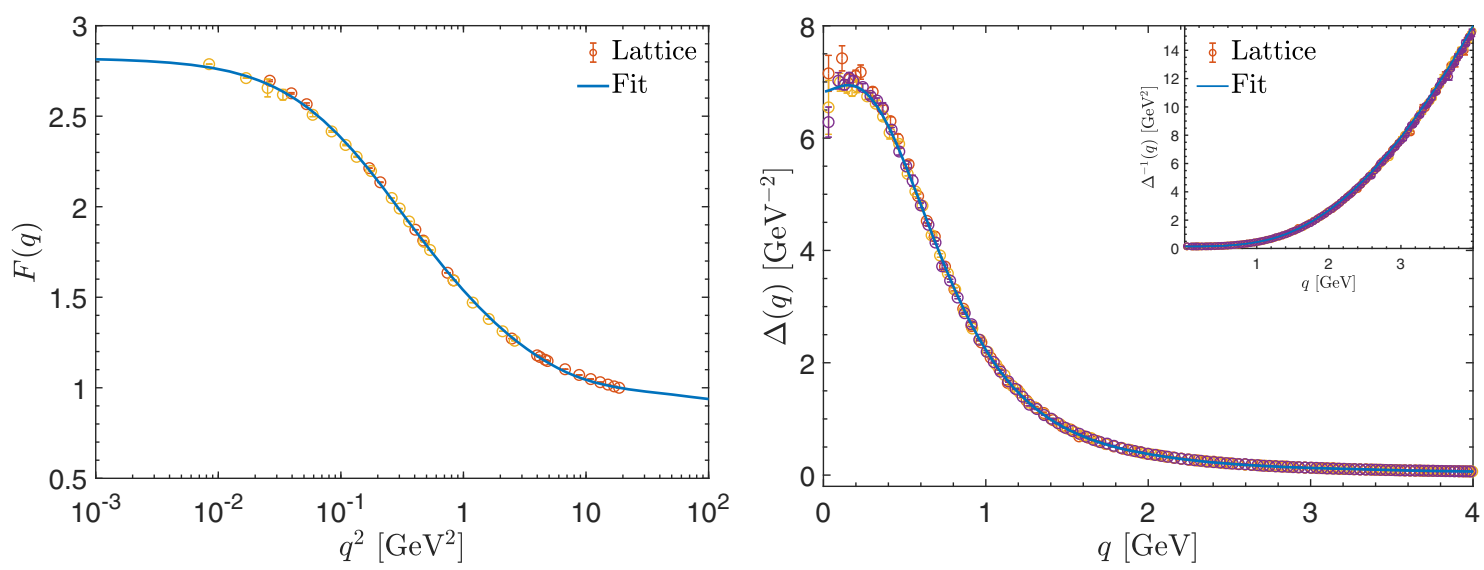

FIG. 3. The fits for $F(q)$ (left panel) and $\Delta(q)$ (right panel) given by Eq. (4.1) and the combination of Eqs. (4.3) and (4.4), respectively (blue continuous curves). In the inset we show the inverse of the gluon propagator, $\Delta^{-1}(q)$. The lattice data are from [70].

\section{NUMERICAL ANALYSIS AND MAIN RESULTS}

In this section we present the numerical analysis and main results for the form factors $X_{1}, X_{2}, X_{3}$, and $X_{10}$, defined by Eq. (3.11).

\section{A. Inputs}

As can be observed from Eq. (3.11), the numerical evaluation of various $X_{i}$ requires the knowledge of the following additional quantities: (i) the ghost dressing function, $F(q)$, (ii) the kinetic part of the gluon propagator, $J(q)$, and (iii) the form factors $A_{1}, A_{3}$, and $A_{4}$ of $H^{\nu \mu}(q, p, r)$. It is important to emphasize that throughout this work, the renormalization point will be fixed at $\mu=4.3 \mathrm{GeV}$, and we will use $\alpha_{s}(\mu) \equiv g^{2} / 4 \pi=0.22$; the determination of this value entails a subtle combination of 4-loop perturbative results, nonperturbative information included in the vacuum condensate of dimension two, and the extraction of $\Lambda_{\mathrm{QCD}}$ from lattice results of the ghostgluon vertex in the Taylor kinematics [118].

In what follows, we will specify the main characteristics of $F(q), J(q)$, and the $A_{i}$, which will be treated as external inputs.

(i) For the ghost dressing function $F(q)$ we employ a physically motivated fit of the solution obtained from the ghost SDE [17], which is in excellent agreement with the lattice data of [70]. Specifically, the fit for $F(q)$ (in Euclidean space) is given by $[84,120]$

$$
\begin{aligned}
F^{-1}(q)= & 1+\frac{9 C_{\mathrm{A}} \alpha_{s}}{48 \pi}\left[1+D \exp \left(-\rho_{4} q^{2}\right)\right] \\
& \times \ln \left(\frac{q^{2}+\rho_{3} M^{2}(q)}{\mu^{2}}\right),
\end{aligned}
$$

where

$$
M^{2}(q)=\frac{m^{2}}{1+q^{2} / \rho_{2}^{2}},
$$

and the fitting parameters are given by $m^{2}=$ $0.16 \mathrm{GeV}^{2}, \quad \rho_{2}^{2}=0.69 \mathrm{GeV}^{2}, \quad \rho_{3}=0.89, \quad \rho_{4}=$ $0.12 \mathrm{GeV}^{-2}$ and $D=2.36$. In the left panel of Fig. 3, we show the lattice data for $F(q)$ together with its corresponding fit given by Eq. (4.1). Clearly, we see that Eq. (4.1) recovers the one-loop result for $F(q)$ for large values of $q^{2}$.

(ii) The way how the quantity $J(q)$ is obtained is considerably more subtle. In particular, as already mentioned at the end of Sec. II, the derivation of $J(q)$ is indirect, in the sense that one first obtains $m^{2}\left(q^{2}\right)$ and then subtracts it from the lattice data for $\Delta^{-1}(q)$ [70], shown in the inset of Fig. 3.

In fact, two different but theoretically equivalent procedures [37] for obtaining the form of $m^{2}\left(q^{2}\right)$ have been developed in the literature: (a) one begins from the general SDE governing $\Delta(q)$, and, through a judicious separation of terms, derives an integral equation for $m^{2}\left(q^{2}\right)$ [36,86], and (b) one solves the BSE responsible for the formation of the bound-state poles $[40,87]$; the corresponding wave function is known to coincide with the first derivative of $m^{2}\left(q^{2}\right)$ [87], from which $m^{2}\left(q^{2}\right)$ may be computed through simple numerical integration. In practice, due to the approximations implemented [37,40], these two methods yield very similar, but not identical results for $m^{2}\left(q^{2}\right)$; the common feature of all solutions is that they are positive definite and monotonically decreasing, displaying the characteristic power law running in the ultraviolet.

In particular, the functional form of $m^{2}\left(q^{2}\right)$ can be accurately represented as [41]

$$
m^{2}\left(q^{2}\right)=\frac{m_{0}^{2}}{1+\left(q^{2} / \rho_{m}^{2}\right)^{1+\gamma}},
$$

where $m_{0}^{2}=0.147 \mathrm{GeV}^{2}$ and the values of $\rho_{m}^{2}$ and $\gamma$ vary depending on the truncations employed. For the 

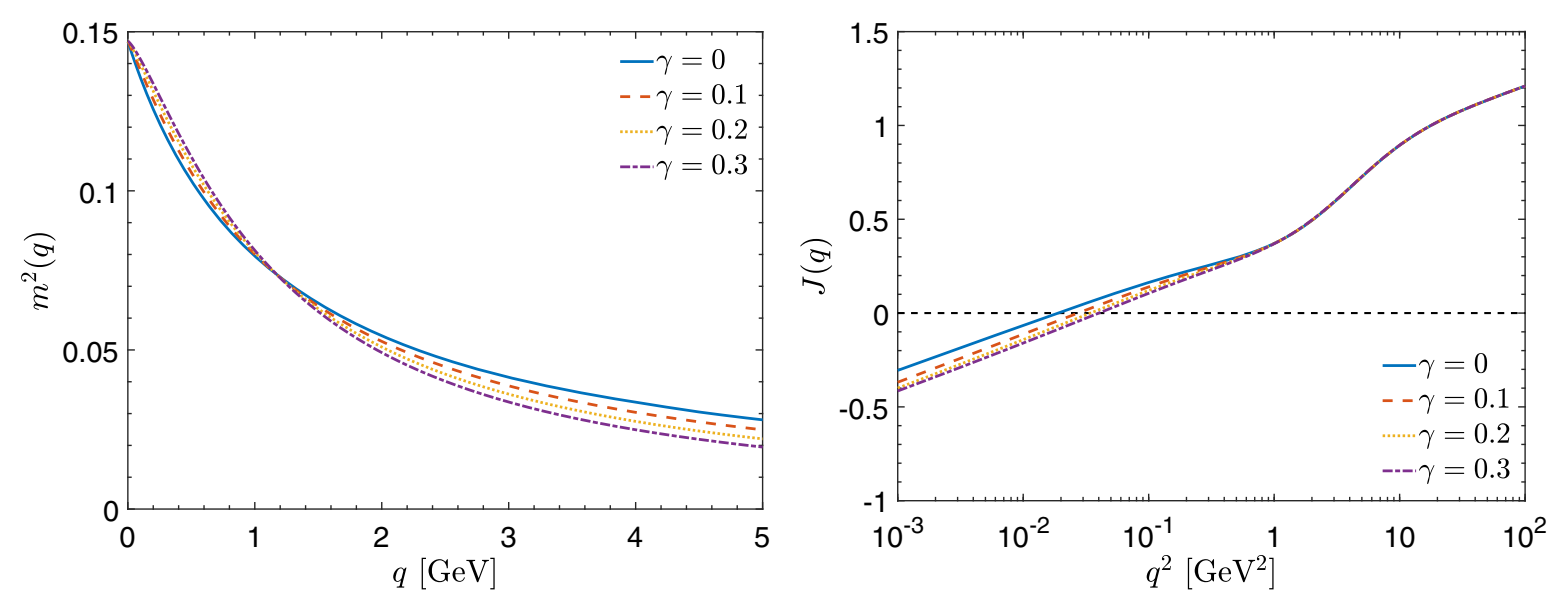

FIG. 4. The dynamical gluon mass, $m^{2}(q)$ given by Eq. (4.3) (left panel), and the corresponding kinetic term $J(q)$ given by Eq. (4.4) (right panel). The curves were obtained for values of $\gamma$ in the range [0, 0.3].

purposes of the present article, we will consider that $\rho_{m}^{2}=1.18 \mathrm{GeV}^{2}$ and we will vary $\gamma$ in the range $[0,0.3]$.

In the left panel of Fig. 4, we plot Eq. (4.3) for different values of $\gamma$ in the range [0,0.3], while in the right panel, we show the corresponding $J(q)$, obtained after subtracting the $m^{2}(q)$ given by Eq. (4.3) from the lattice data $\Delta^{-1}(q)$, according to Eq. (2.6). All curves for $J(q)$ may be parametrized by the same functional form

$$
\begin{aligned}
J(q)= & 1+\frac{C_{\mathrm{A}} \alpha_{s}}{4 \pi}\left(1+\frac{\tau_{1}}{q^{2}+\tau_{2}}\right) \\
& \times\left[2 \ln \left(\frac{q^{2}+\rho m^{2}(q)}{\mu^{2}}\right)+\frac{1}{6} \ln \left(\frac{q^{2}}{\mu^{2}}\right)\right],
\end{aligned}
$$

where the $\gamma$ dependence of $\rho \equiv \rho(\gamma), \tau_{1} \equiv \tau_{1}(\gamma)$ and $\tau_{2} \equiv \tau_{2}(\gamma)$ has been suppressed for compactness. For values of $\gamma$ in the range [0,0.3], these functions can be represented by

$$
\begin{aligned}
\rho(\gamma) & =100.8-82.21 \gamma^{1.28}, \\
\tau_{1}(\gamma) & =9.87-6.96 \gamma \\
\tau_{2}(\gamma) & =0.80+0.11 \exp (-10 \gamma) .
\end{aligned}
$$

In Figs. 5, we show fits for the functions $\rho(\gamma)$, $\tau_{1}(\gamma)$, and $\tau_{2}(\gamma)$; the values employed for obtaining these curves are marked with stars.

In addition, notice that Eqs. (4.3) and (4.4) not only reproduce, by construction, the curve for $\Delta(q)$ shown in the right panel of Fig. 3, but also incorporate the following crucial features [18]: (a) the infrared finiteness of the gluon propagator; (b) the presence of "protected" and "unprotected" logarithms, i.e., $\ln \left(q^{2}+m^{2}\right)$ and $\ln \left(q^{2}\right)$, originating, respectively from the gluon and ghost loops of the SDE for $\Delta(q)$; (c) the massless ghost logarithms force $J(q)$ to reverse sign and diverge logarithmically in the infrared, with a zero-crossing around the region of a few hundred $\mathrm{MeV}$.

In the right panel of Fig. 4 one may appreciate that the precise location of the zero-crossing, $q_{0}^{\mathrm{J}}$, depends
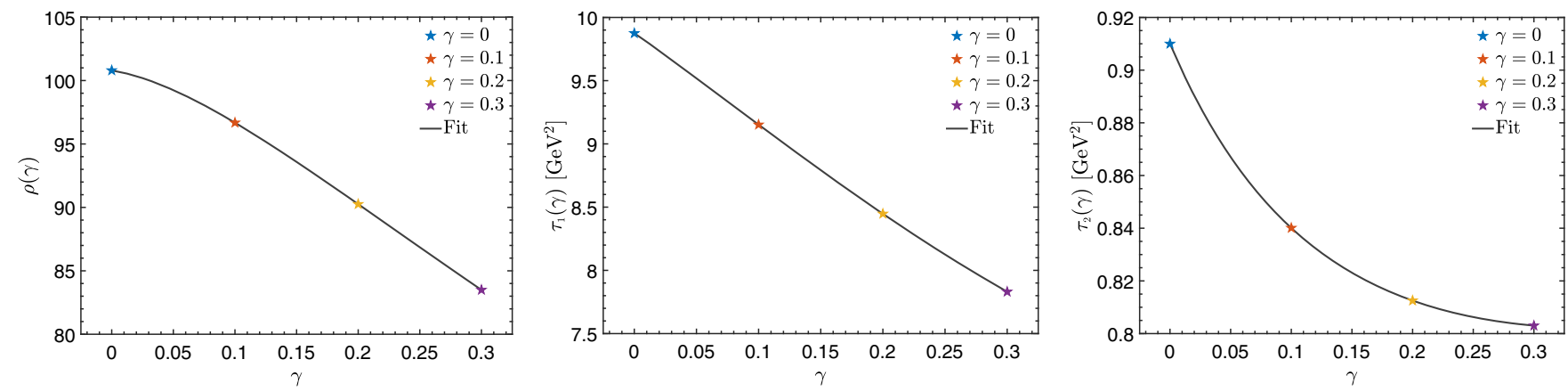

FIG. 5. The functions $\rho(\gamma)$ (left), $\tau_{1}(\gamma)$ (center), and $\tau_{2}(\gamma)$ (right) appearing in the Eq. (4.4); their functional forms are given in Eq. (4.5). The stars represent the following values for the set $\left[\gamma, \rho, \tau_{1}\left(\mathrm{in} \mathrm{GeV}^{2}\right), \tau_{2}\left(\mathrm{in} \mathrm{GeV}^{2}\right)\right]:[0,100.8,9.87,0.91]$ (blue stars), [0.1, 96.7, 9.15, 0.84] (red stars), [0.2, 90.3, 8.45, 0.81] (yellow stars), and [0.3, 83.5, 7.84, 0.80] (purple stars). 

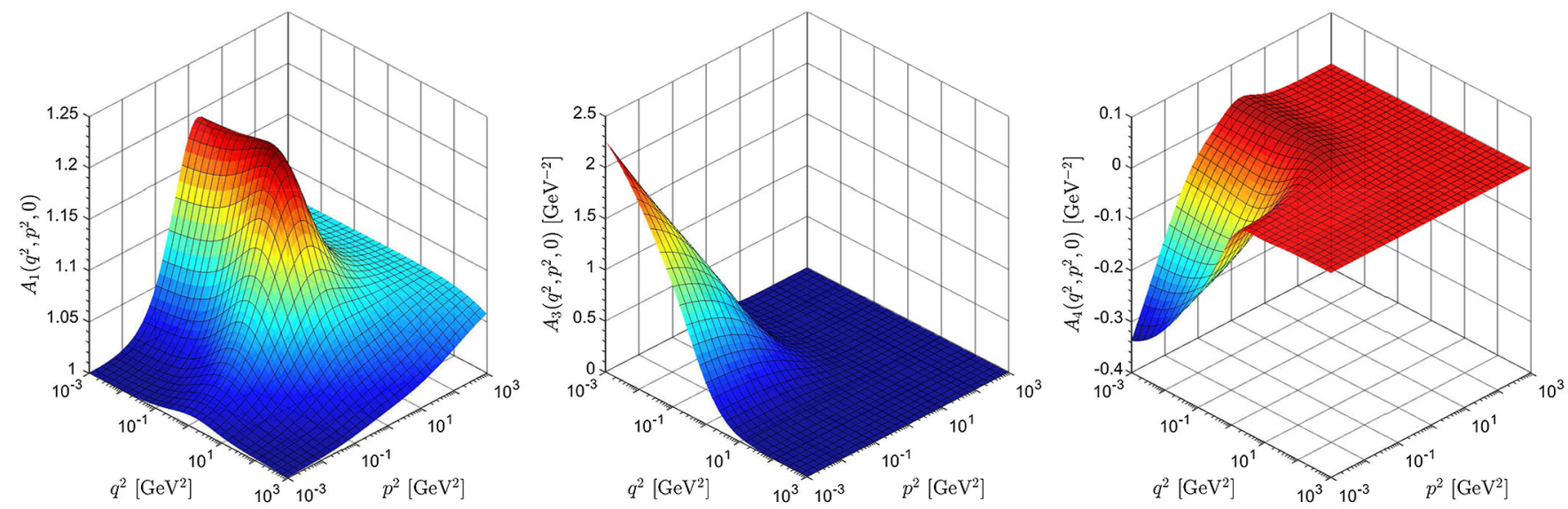

FIG. 6. The form factors of the ghost-gluon scattering kernel $A_{1}\left(q^{2}, p^{2}, \phi\right)$ (first panel), $A_{3}\left(q^{2}, p^{2}, \phi\right)$ (second panel), and $A_{4}\left(q^{2}, p^{2}, \phi\right)$ (third panel) for $\phi=0$ and $\alpha_{s}=0.22$.

on the power $\gamma$, which controls the functional form of $m^{2}(q)$ in Eq. (4.3). More specifically, we have the following values for the pair $\left[\gamma, q_{0}^{\mathrm{J}}(\right.$ in $\left.\mathrm{MeV})\right]$ : $[0,140]$ (blue continuous), $[0.1,166]$ (red dashed), $[0.2,187]$ (yellow dotted), and [0.3, 202] (purple dash-dotted).

(iii) The final ingredients needed for the evaluation of the $\mathrm{BC}$ solution are the form factors $A_{1}, A_{3}$, and $A_{4}$ of the ghost-gluon kernel $H_{\nu \mu}(q, p, r)$, defined in Eq. (2.5). Their nonperturbative evaluation for general Euclidean momenta has been presented in [84], where the one-loop dressed version of the SDE satisfied by $H_{\nu \mu}(q, p, r)$ was employed. In Fig. 6 we show a representative case for $A_{1}, A_{3}$, and $A_{4}$, when the angle between the momenta $q$ and $p$ is fixed at $\phi=0$. Note in particular that $A_{1}$ is finite within the entire range of its momenta, whereas $A_{3}$ and $A_{4}$ display a logarithmic divergence in the deep infrared.

\section{B. The three-gluon form factors: General kinematics}

With the inputs introduced in the previous subsection, the form factors $X_{1}, X_{2}, X_{3}$, and $X_{10}$ may now be computed from Eqs. (3.11).

The evaluation of Eqs. (3.11) is carried out for general Euclidean kinematics; we will express the form factors as functions of $q^{2}, r^{2}$, and the angle $\theta$ formed between $q$ and $r$, namely $X_{i}(q, r, p) \rightarrow X_{i}\left(q^{2}, r^{2}, \theta\right)$. For the numerical computation of the relevant integrals we use logarithmically spaced grids for $q^{2}$ and $r^{2}$, with 96 values for each, in the range $\left[5 \times 10^{-5} \mathrm{GeV}^{2}, 10^{4}, \mathrm{GeV}^{2}\right]$. The corresponding grid for the angle $\theta$ is uniformly spaced, with 19 values distributed within $[0, \pi]$. Moreover, the required interpolations of the results for $A_{1}, A_{3}$, and $A_{4}$, obtained in [84], are performed using tensor products of B-splines [121].

The results for $X_{1}\left(q^{2}, r^{2}, \theta\right), X_{2}\left(q^{2}, r^{2}, \theta\right), X_{3}\left(q^{2}, r^{2}, \theta\right)$, and $X_{10}\left(q^{2}, r^{2}, \theta\right)$ are shown in Figs. 7-10, respectively.
In each of these figures, we present the corresponding form factor for three representative values of the angle: $\theta=0$ (top left panels), $\theta=\pi / 3$ (top right panels), and $\theta=2 \pi / 3$ (bottom left panels). In addition, we provide a visual impression of the impact that the ghost sector has on the $X_{i}$ by plotting the corresponding "Abelianized" quantities, $\hat{X}_{i}$, in the bottom right panels; these latter quantities are given by Eq. (3.13), and are independent of the angle $\theta$. Since $\hat{X}_{10}=0$, we occupy its panel in Fig. 10 with one additional configuration, namely $X_{10}\left(q^{2}, r^{2}, \theta=\pi / 2\right)$.

The results exhibit the following features: (i) in the infrared, $X_{1}, X_{2}$, and $X_{3}$ depart considerably from their tree level values $\left(1,0\right.$, and 0 , respectively), while $X_{10}$, even though nonvanishing, is very suppressed; (ii) in the ultraviolet, all form factors approach their expected perturbative behavior; (iii) the patterns displayed by the $X_{i}$ are rather similar to those of the $\hat{X}_{i}$, but with small "oscillations" distributed around their main structures, owing to the contributions from the ghost sector; (iv) in general, they display a mild dependence on the angle $\theta$.

It is important to emphasize that, while the form factors $X_{1}, X_{2}$, and $X_{10}$ diverge at most logarithmically in the infrared, under certain special kinematic circumstances $X_{3}$ displays a pole divergence. This, in turn, is the reason for employing double-log graphs for the surfaces shown in Fig. 9.

To appreciate this point, note first that, due to the presence of the factor $\left(q^{2}-r^{2}\right)$ in the denominator of Eq. (3.11), the computation of $X_{3}$ in the limit $q^{2} \rightarrow r^{2}:=$ $Q^{2}$ requires the use of a limiting procedure, which amounts to taking appropriate total or partial derivatives. Note that the equality $q^{2}=r^{2}$ may be realized for any value of the angle $\theta$; momentum conservation restricts $p^{2}$ to satisfy

$$
p^{2}=2 Q^{2}(1+\cos \theta)
$$

with limiting cases $p^{2}=0$ and $p^{2}=4 Q^{2}$. 

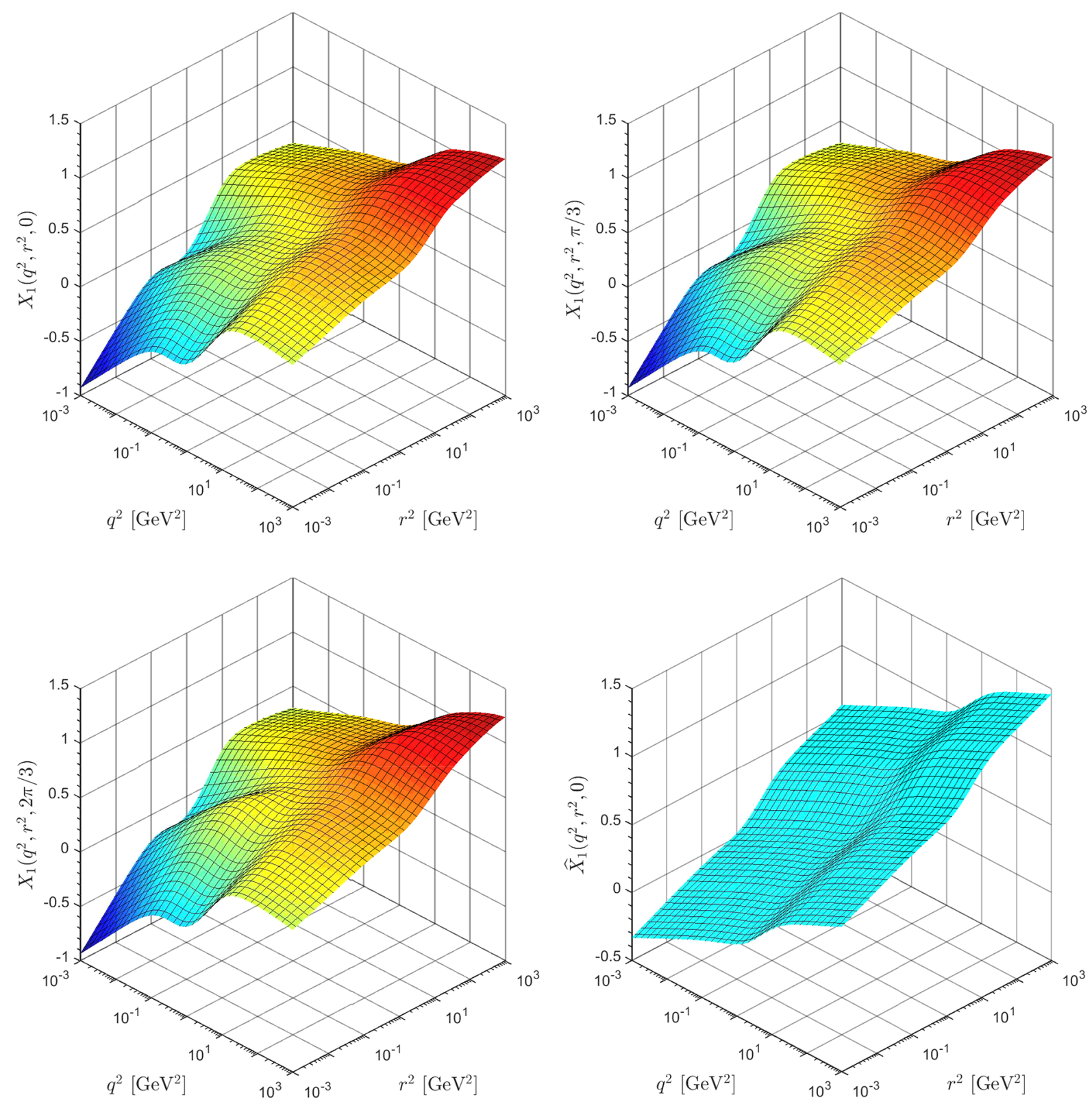

FIG. 7. $X_{1}\left(q^{2}, r^{2}, \theta\right)$ for $\theta=0$ (top left), $\pi / 3$ (top right), and $2 \pi / 3$ (bottom left), together with the "Abelianized" $\hat{X}_{1}$ (bottom right).

In order to simplify the algebra without compromising the essence, let us revert to the "Abelianized" form of $X_{3}$ given in Eq. (3.13), and let $q^{2} \rightarrow r^{2}:=Q^{2}$. Then, one has that in Euclidean space

$$
\hat{X}_{3}(Q)=-\frac{d J(Q)}{d Q^{2}}
$$

which, after employing the functional form for $J(Q)$ given in Eq. (4.4), reads

$$
\begin{aligned}
\hat{X}_{3}(Q)= & -\frac{C_{\mathrm{A}} \alpha_{s}}{24 \pi}\left[\frac{1}{Q^{2}}\left(1+\frac{\tau_{1}}{Q^{2}+\tau_{2}}\right)-\frac{\tau_{1}}{\left(Q^{2}+\tau_{2}\right)^{2}} \ln \left(\frac{Q^{2}}{\mu^{2}}\right)\right] \\
& +\cdots ;
\end{aligned}
$$

evidently, the above expression contains a simple pole together with a subleading logarithmic divergence, while the ellipses denote terms that are finite as $Q^{2} \rightarrow 0$.

Regarding the behavior of $\hat{X}_{3}(Q)$ observed above, the following remarks are in order.

(a) Note that this particular divergence is not an artifact of the $\mathrm{BC}$ basis, which in [54] was advocated to be free of kinematic singularities. In fact, it should be clear that the origin of the divergence is dynamical, stemming from the presence of the "unprotected" logarithm, and hence, from the nonperturbative masslessness of the ghost. If for instance the aforementioned logarithm had been omitted from the $J(Q)$, the answer in Eq. (4.8) would be perfectly finite; and the same 

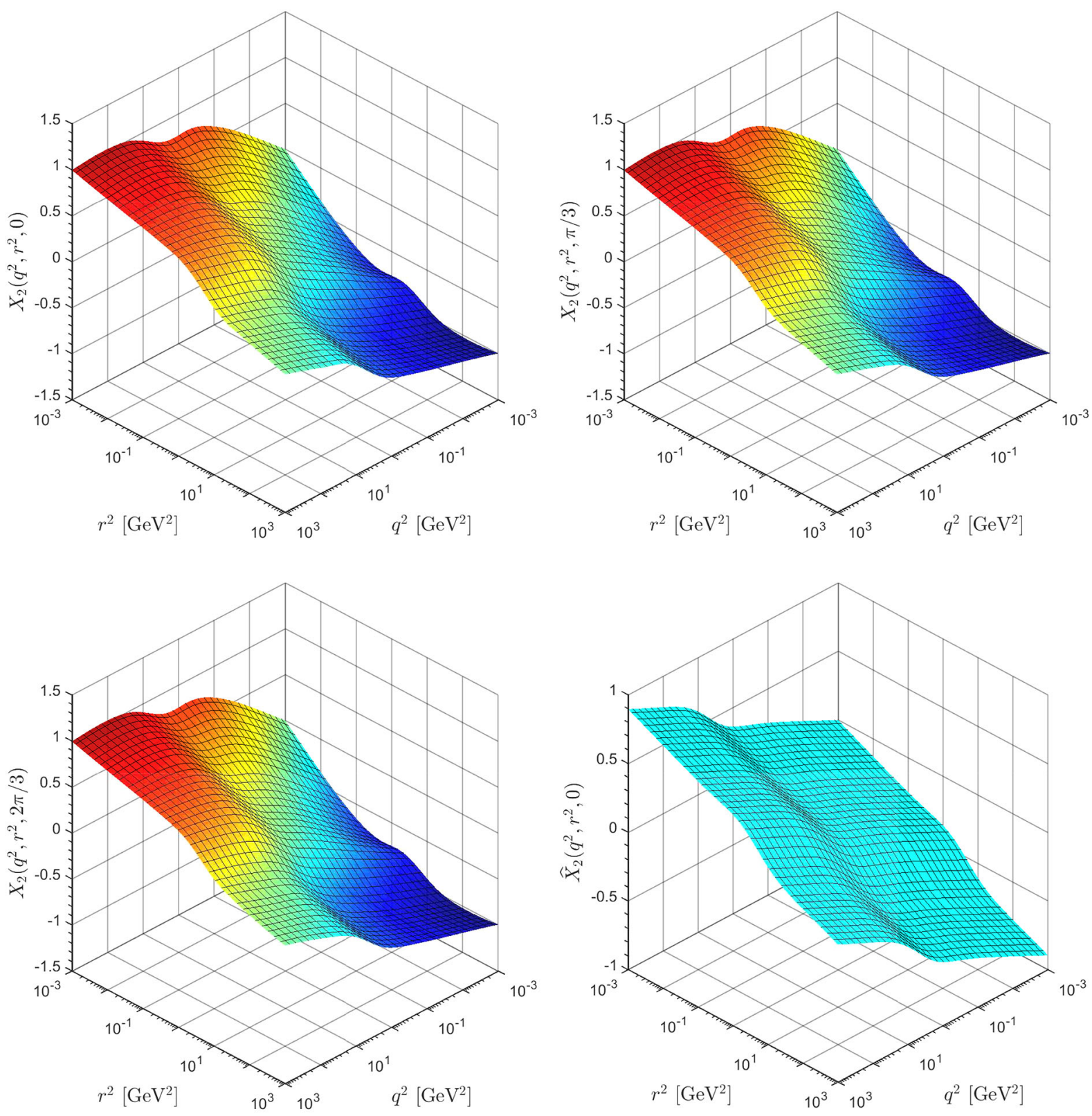

FIG. 8. $X_{2}\left(q^{2}, r^{2}, \theta\right)$ for $\theta=0$ (top left), $\pi / 3$ (top right), and $2 \pi / 3$ (bottom left), together with the "Abelianized" $\hat{X}_{2}$ (bottom right). Note that, in order to better visualize the surfaces, the $q^{2}$ and $r^{2}$ axes have been rotated by $\pi / 2$ with respect to the other 3D figures.

would be true if the argument of the logarithm had been saturated by a "ghost mass," whose generation, however, does not occur dynamically.

(b) The type of pole divergence found in Eq. (4.8) should be clearly distinguished from those appearing in $V_{\alpha \mu \nu}(q, r, p)$. Note, in particular, that the pole $1 / q^{2}$ (or any other) is explicitly present, and leads to a divergence when $q^{2} \rightarrow 0$, while $r^{2}=p^{2} \neq 0$. In fact, unlike $V_{\alpha \mu \nu}(q, r, p)$, the direct substitution of $q^{2}=0$ into the $\hat{X}_{3}$ of Eq. (3.13) yields simply $\hat{X}_{3}\left(r^{2}\right)=$ $-\frac{[J(r)-J(0)]}{r^{2}}$ (in Euclidean space), which is only logarithmically divergent [due to $J(0)]$ as long as $r^{2} \neq 0$. (c) The above arguments hold also for the full (nonAbelian) $X_{3}(q, r, p)$, given that $F(q)$ is completely finite, while the $A_{i}(q, p, r)$ are at most logarithmically divergent in the infrared, as was found in [84].

(d) A concrete manifestation of the divergences captured by Eq. (4.8) will be encountered shortly in Sec. IV D, in the context of the "totally symmetric" and "asymmetric" configurations, which fulfill the kinematic circumstances described above, being both special cases of Eq. (4.6), for $\theta=2 \pi / 3$ and $\theta=\pi$, respectively.

Let us next turn to $X_{10}$; this particular form factor vanishes identically at both tree and one-loop 

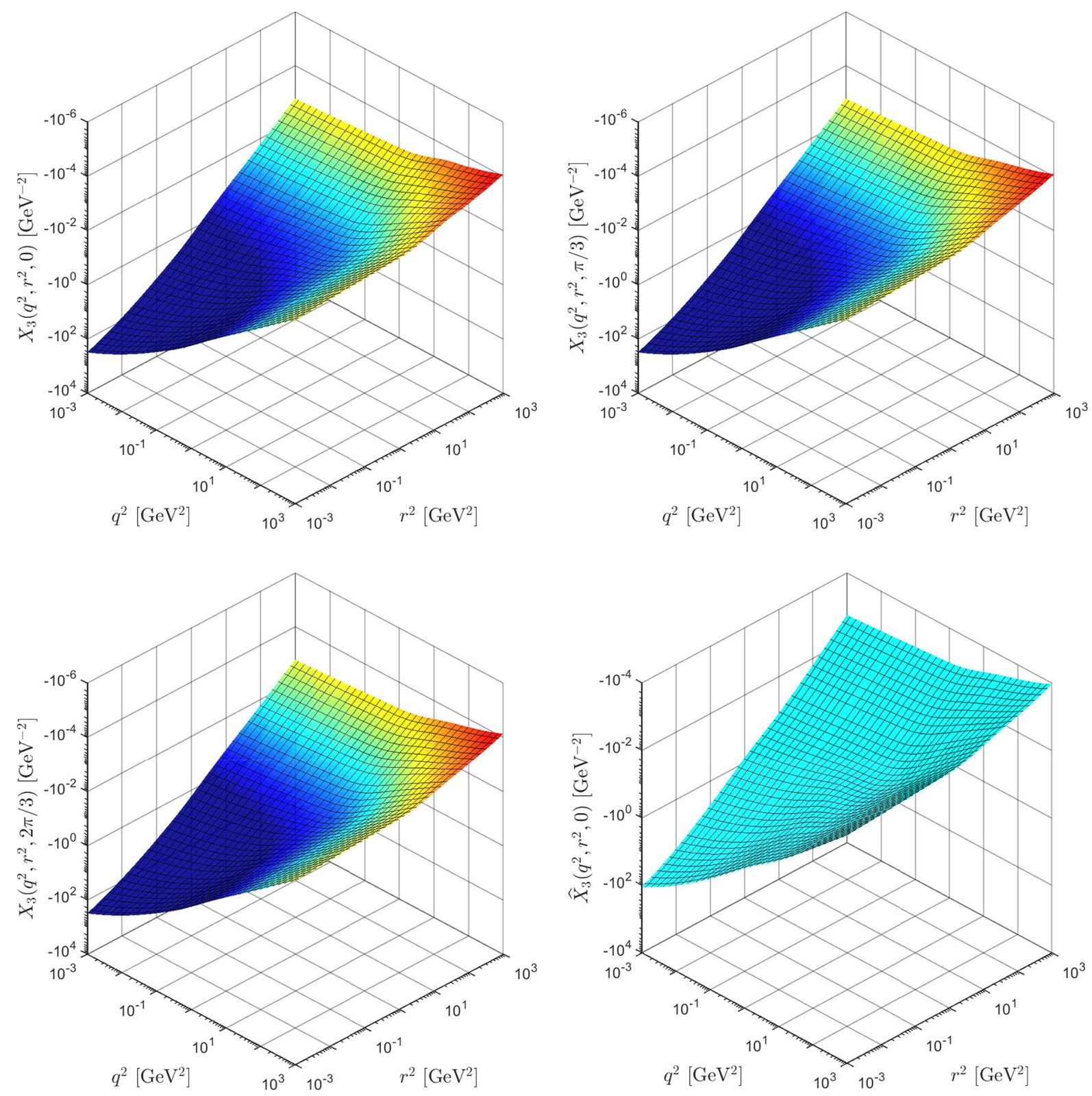

FIG. 9. $X_{3}\left(q^{2}, r^{2}, 0\right)$ for $\theta=0$ (top left), $\pi / 3$ (top right), and $2 \pi / 3$ (bottom left), together with the "Abelianized" $\hat{X}_{3}$ (bottom right).

levels [88]. As we can see in Fig. 10, $X_{10}\left(q^{2}, p^{2}, \theta\right)$ does not vanish nonperturbatively; note, however, that it is extremely suppressed in comparison with the other $X_{i}$, tending rapidly to zero whenever any of its momenta becomes large.

We end this subsection by comparing the infrared suppression of the nonperturbative $X_{1}$ with that obtained from a direct one-loop calculation. Specifically, in the left panel of Fig. 11 we compare our result for $X_{1}\left(q^{2}, r^{2}, \pi / 2\right)$ (colored surface) with the corresponding one-loop expression, given by Eq. (A8) (cyan surface). Evidently, the colored surface is considerably more "tilted" toward the infrared region, due to the presence of the zero crossing. It is also interesting to observe that $X_{1}$ reaches its maximum value along the curve projected on the "diagonal" plane, ${ }^{4}$ where $q^{2}=r^{2}$, and then drops in all directions. To appreciate this effect more clearly, in the right panel of Fig. 11 we selected three additional slices of the 3D plot; indeed, the symmetric limit corresponds to the highest kinematic configuration of $X_{1}\left(q^{2}, r^{2}, \pi / 2\right)$.

\footnotetext{
${ }^{4}$ The one-loop expression for this slice is given by Eq. (A10).
} 

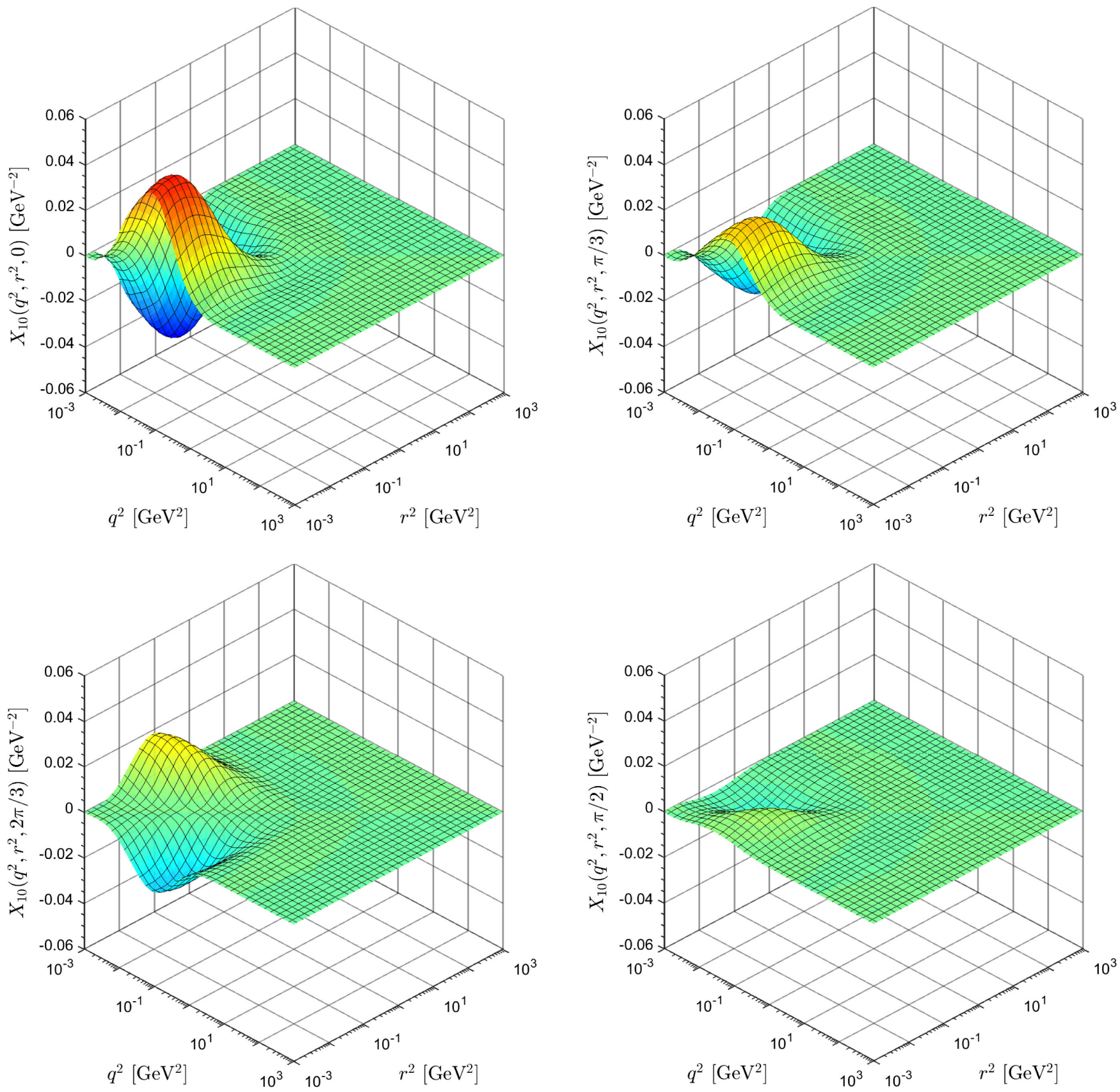

FIG. 10. $X_{10}\left(q^{2}, r^{2}, \theta\right)$ for $\theta=0$ (top left), $\pi / 3$ (top right), $2 \pi / 3$ (bottom left), and $\pi / 2$ (bottom right).

\section{Evaluation of the remaining form factors: An example}

In principle, if the functional dependence of $X_{1}, X_{2}$, and $X_{3}$ on $q, r$, and $p$ is known, the remaining $X_{i}$ can be obtained by invoking the Bose symmetry relations of Eqs. (3.8); for instance, $X_{4}(q, r, p)$ can be obtained by permuting the arguments of $X_{1}(q, r, p)$ to $X_{1}(r, p, q)$. In practice, however, what one has is the values of $X_{1}(q, r, p)$ tabulated for a grid of points for $q^{2}, r^{2}$, and $\theta$ (the angle between $q$ and $r$, which we represent as $X_{1}\left(q^{2}, r^{2}, \theta\right)$. In order to evaluate the data point $X_{4}\left(q^{2}, r^{2}, \theta\right)=X_{1}\left(r^{2}, p^{2}, \varphi\right)$, where $\varphi$ is the angle between $r$ and $p$, one invokes momentum conservation to relate $p^{2}=q^{2}+r^{2}+2 q \cdot r$. Similarly, one finds for the angle

$\varphi=\cos ^{-1}\left(\frac{r \cdot p}{|r||p|}\right)=\cos ^{-1}\left[-\frac{(|r|+|q| \cos \theta)}{\sqrt{q^{2}+r^{2}+2|q||r| \cos \theta}}\right]$. 

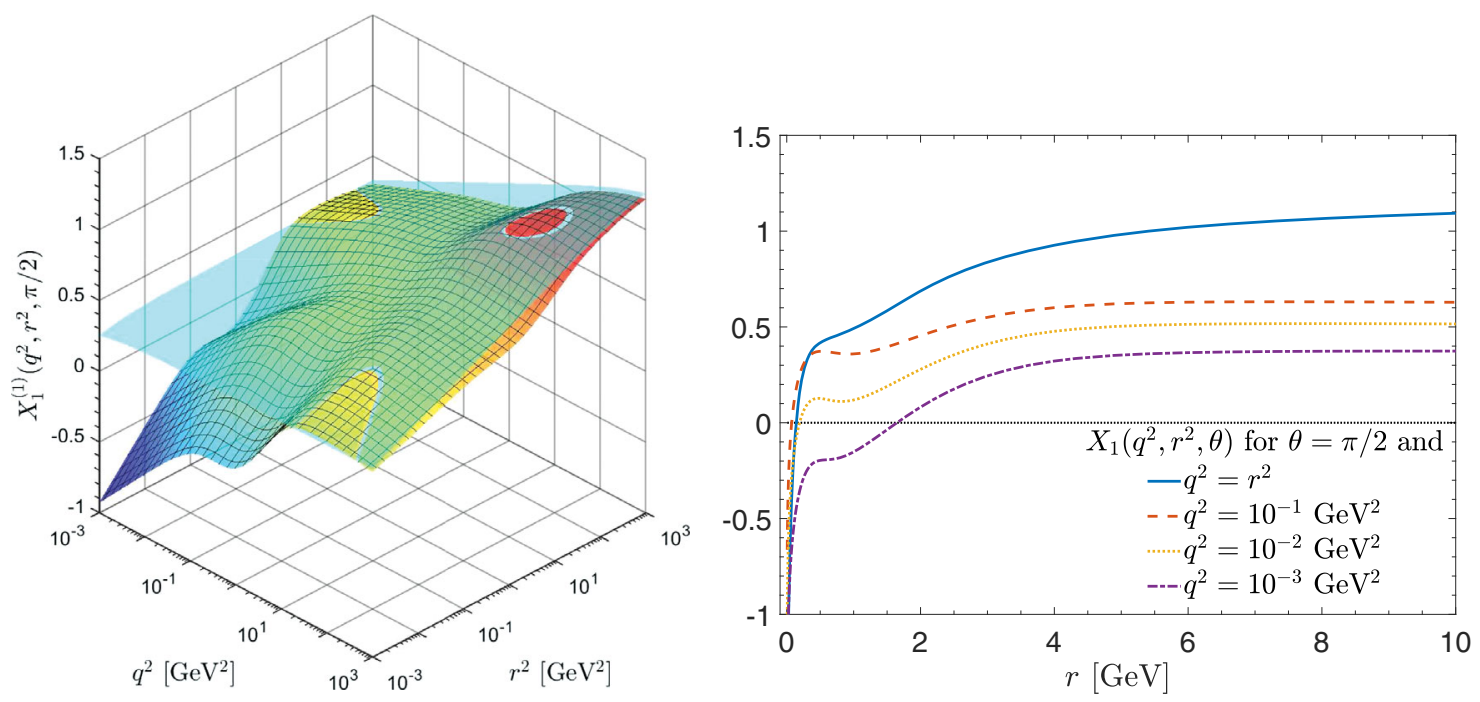

FIG. 11. Comparison of the nonperturbative (colored surface) and the one-loop (cyan surface) results for $X_{1}\left(q^{2}, r^{2}, \pi / 2\right)$ (left panel). Special kinematic limits of $X_{1}$ for a fixed values of $q^{2}$ when $\theta=\pi / 2$ (right panel).

Then, one carries out a three-dimensional interpolation, using, for example, tensor products of B-splines [121], and obtains the value of $X_{1}$ at $\left(r^{2}, p^{2}, \varphi\right)$.

In Fig. 12 we show the result of the exercise described above for $X_{4}\left(q^{2}, r^{2}, \theta\right)$, for two representative values of $\theta$; notice that $X_{4}$ is not symmetric under the exchange of $q$ and $r$, a fact that is clearly reflected in the shape of the surfaces obtained.

\section{Special kinematics limits}

In this subsection we extract two special kinematic configurations from the general solutions for $X_{i}$ reported

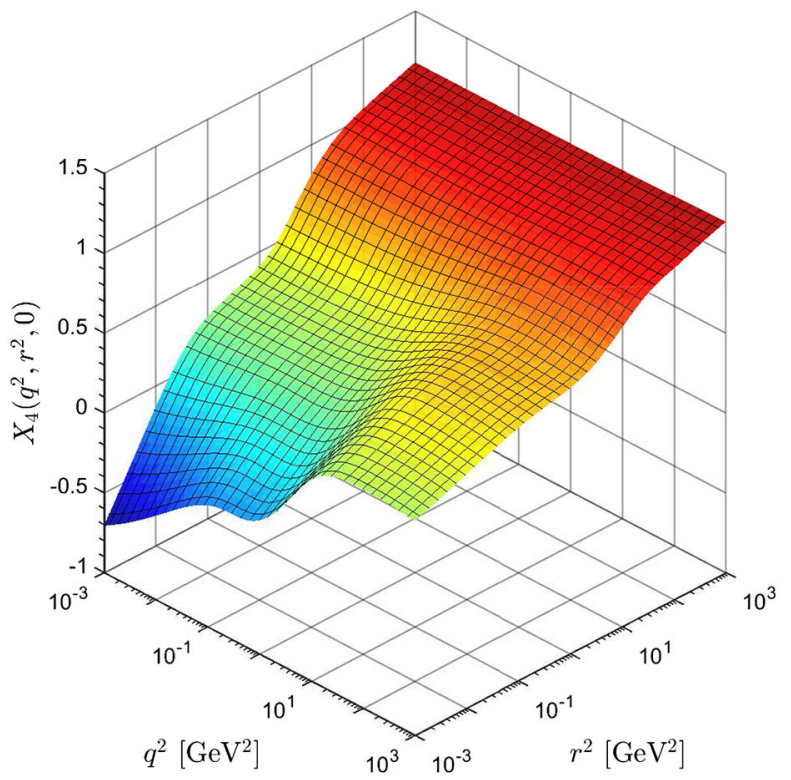

above, and compare them with the corresponding one-loop results, given in Appendix A.

(i) First we consider the totally symmetric limit, obtained when

$$
\begin{aligned}
q^{2}=p^{2}=r^{2}=Q^{2}, \quad q \cdot p & =q \cdot r=p \cdot r=-\frac{1}{2} Q^{2}, \\
\theta & =2 \pi / 3 ;
\end{aligned}
$$

the form factors in this configuration will be denoted by $X_{i}(Q)$.

Recalling that $X_{2}$ and $X_{10}$ are antisymmetric under the exchange of at least two of their arguments [see Eq. (3.7)],

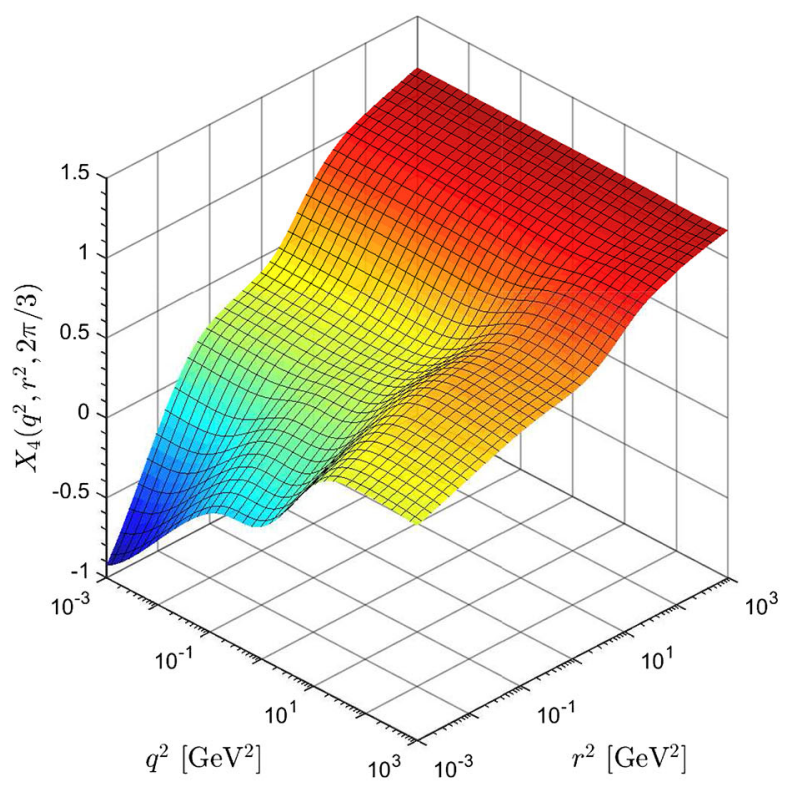

FIG. 12. The form factor $X_{4}\left(q^{2}, r^{2}, \theta\right)$ for $\theta=0$ (left) and $2 \pi / 3$ (right) obtained from $X_{1}\left(r^{2}, p^{2}, \varphi\right)$ using the Eq. (4.9). 

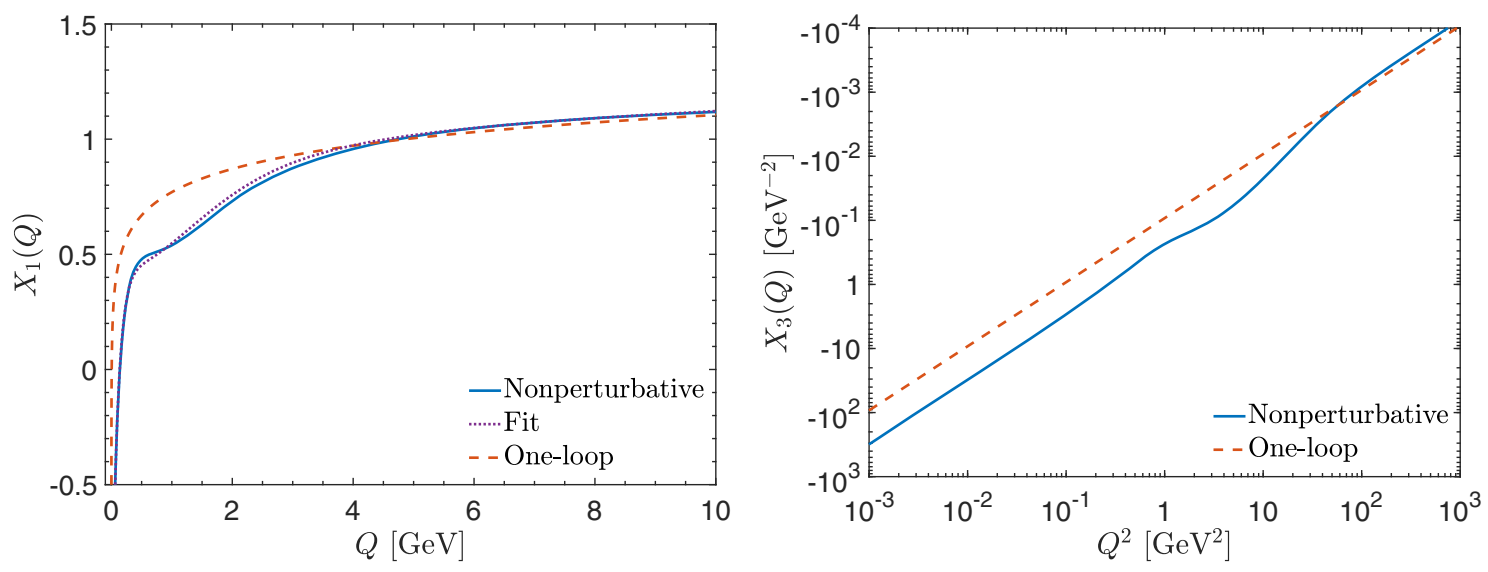

FIG. 13. Comparison of the nonperturbative form factors $X_{1}(Q)$ (left panel) and $X_{3}(Q)$ (right panel) with their one-loop counterparts, given by Eqs. (A3) (red dashed), in the totally symmetric limit. In the left panel we also plot the fit for $X_{1}(Q)$ given by Eq. (4.11) with $\gamma=0$ (purple dotted).

it is clear that, in this particular configuration, they both vanish identically. Therefore, we consider only $X_{1}(Q)$ and $X_{3}(Q)$, which may be obtained as a projection on the plane $q^{2}=r^{2}$ of their 3D surfaces, shown on the bottom left sides of Figs. 7 and 9, respectively.

In the left panel of Fig. 13, we can see that $X_{1}(Q)$ displays a notable discrepancy from its one-loop behavior [see Eq. (A3)] in the window of momenta $0.5 \mathrm{GeV} \leq Q \leq$ $3.0 \mathrm{GeV}$. In that range, $X_{1}(Q)$ suffers an abrupt change of curvature, which, at first sight, might be considered as a numerical artifact. However, as we will see in Sec. V B, this "bending" is crucial for reproducing a characteristic "knee" that appears in the lattice data in the same region of momenta.

In the same figure we also show a physically motivated fit for $X_{1}(Q)$ (purple dotted line), which is in good agreement with our nonperturbative result and recovers the one-loop result in the ultraviolet. Specifically,

$$
\begin{aligned}
X_{1}(Q)= & 1+\frac{C_{\mathrm{A}} \alpha_{s}}{96 \pi}\left[1+\frac{\kappa_{1}}{1+\left(Q^{2} / \kappa_{2}\right)^{1+\kappa_{3}}}\right] \\
& \times\left\{33 \ln \left[\frac{Q^{2}+\rho_{\ell} m^{2}\left(Q^{2}\right)}{\mu^{2}}\right]+\ln \left(\frac{Q^{2}}{\mu^{2}}\right)\right\} \\
& +\frac{C_{\mathrm{A}} \alpha_{s}}{16 \pi}(1-\mathrm{I}),
\end{aligned}
$$

where $m^{2}\left(Q^{2}\right)$ is given by Eq. (4.3) and the corresponding value of $\gamma$ should be used, while I is given in Eq. (A4). The fitting parameters for the case where $\gamma=0$ are $\kappa_{1}=135.3$, $\kappa_{2}=0.086 \mathrm{GeV}^{2}, \kappa_{3}=0$, and $\rho_{\ell}=140.4$.

As for $X_{3}(Q)$, the present kinematic limit is precisely of the type considered in Sec. IV B, leading to Eq. (4.8); note that the substitution of $\theta=2 \pi / 3$ into Eq. (4.6) yields indeed $p^{2}=Q^{2}$. Given that both $X_{3}(Q)$ and its perturbative counterpart [see Eq. (A3)] diverge as $1 / Q^{2}$ in the infrared, they are displayed in the log-log plot shown on the right panel of Fig. 13. The coincidence with the perturbative result is quite satisfactory in the ultraviolet, but a considerable departure is observed as one moves toward the infrared, where the two curves run nearly "parallel" to each other, with the nonperturbative $X_{3}(Q)$ (blue curve) being about a factor of 4-5 larger. Evidently, even though both curves are dominated by the pole $1 / Q^{2}$, the values of their corresponding residues are rather different.

(ii) The asymmetric limit, defined when

$$
p=0, \quad r=-q, \quad \theta=\pi
$$

in what follows we will express our results for this configuration in terms of the momentum $q$, i.e., $X_{i}\left(q^{2}, q^{2}, \pi\right)$. In this configuration, the tensorial structure of $\Gamma_{\mathrm{L}}^{\alpha \mu \nu}(q, r, p)$ reduces to that given in Eq. (A6).

In Fig. 14 we show $X_{1}^{(1)}\left(q^{2}, q^{2}, \pi\right)$ (left panel) and $X_{3}^{(1)}\left(q^{2}, q^{2}, \pi\right)$ (right panel), which are clearly very similar to those obtained in the symmetric limit. More specifically, $X_{1}^{(1)}\left(q^{2}, q^{2}, \pi\right)$ deviates mildly from its one-loop behavior, displaying the characteristic bending in the same range of momenta, while $X_{3}^{(1)}\left(q^{2}, q^{2}, \pi\right)$ diverges again as a pole, corresponding to the case where, for $\theta=\pi$, Eq. (4.6) yields $p^{2}=0$. As expected, in the ultraviolet regime both form factors tend towards the behavior predicted by the one-loop result given in Eq. (A7).

\section{COMPARISON WITH PREVIOUS RESULTS}

In this section we present a direct comparison between our results and those obtained from (i) the SDE analysis of [14,83], and (ii) the lattice simulation of [25].

For the purposes of this section, it is convenient to introduce the following transversely projected counterparts of $\boldsymbol{\Gamma}_{\alpha \mu \nu}(q, r, p), \Gamma_{\alpha \mu \nu}(q, r, p)$, and $\Gamma_{\alpha \mu \nu}^{(0)}(q, r, p)$, defined as 

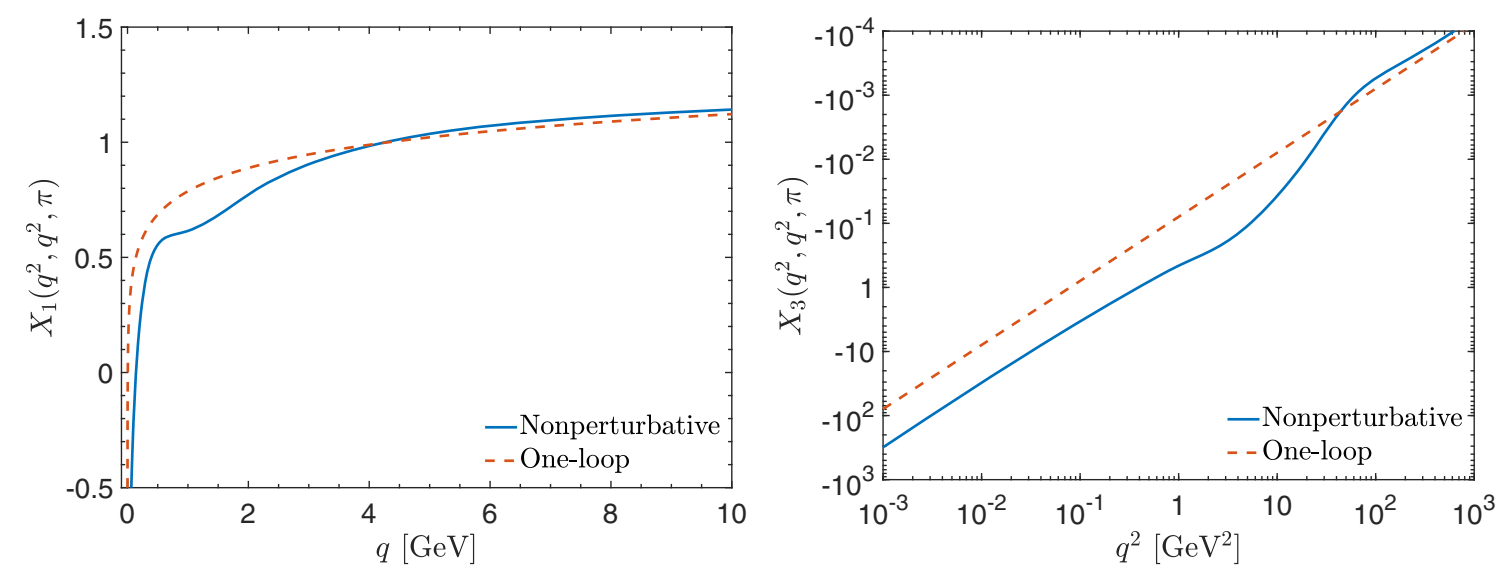

FIG. 14. Comparison of the nonperturbative form factors $X_{1}^{(1)}\left(q^{2}, q^{2}, \pi\right)$ (left panel) and $X_{3}^{(1)}\left(q^{2}, q^{2}, \pi\right)$ (right panel) with their oneloop counterparts given by Eqs. (A7) (red dashed) in the asymmetric limit.

$$
\begin{aligned}
\bar{\Gamma}_{\alpha \mu \nu}(q, r, p) & :=P_{\alpha^{\prime} \alpha}(q) P_{\mu^{\prime} \mu}(r) P_{\nu^{\prime} \nu}(p) \mathbb{\Gamma}^{\alpha^{\prime} \mu^{\prime} \nu^{\prime}}(q, r, p), \\
\bar{\Gamma}_{\alpha \mu \nu}(q, r, p) & :=P_{\alpha^{\prime} \alpha}(q) P_{\mu^{\prime} \mu}(r) P_{\nu^{\prime} \nu}(p) \Gamma^{\alpha^{\prime} \mu^{\prime} \nu^{\prime}}(q, r, p), \\
\bar{\Gamma}_{\alpha \mu \nu}^{(0)}(q, r, p) & :=P_{\alpha^{\prime} \alpha}(q) P_{\mu^{\prime} \mu}(r) P_{\nu^{\prime} \nu}(p) \Gamma^{(0) \alpha^{\prime} \mu^{\prime} \nu^{\prime}}(q, r, p) .
\end{aligned}
$$

Note that, by virtue of Eq. (2.9), we have the important relation

$$
\overline{\boldsymbol{\Gamma}}_{\alpha \mu \nu}(q, r, p)=\bar{\Gamma}_{\alpha \mu \nu}(q, r, p) .
$$

Next we introduce the general projector $L(q, r, p)$, given by

$$
\begin{aligned}
L(q, r, p) & =\frac{W^{\alpha \mu \nu}(q, r, p) \overline{\boldsymbol{\Gamma}}_{\alpha \mu \nu}(q, r, p)}{W^{\alpha \mu \nu}(q, r, p) W_{\alpha \mu \nu}(q, r, p)} \\
& =\frac{W^{\alpha \mu \nu}(q, r, p) \bar{\Gamma}_{\alpha \mu \nu}(q, r, p)}{W^{\alpha \mu \nu}(q, r, p) W_{\alpha \mu \nu}(q, r, p)},
\end{aligned}
$$

where in the second step we have used Eq. (5.2). The precise form of the tensor $W^{\alpha \mu \nu}(q, r, p)$ will depend on the particular circumstances considered.

\section{A. Comparison with SDE-derived results}

Next, we compare our results with those in $[14,83]$. In that work, an approximate version of the SDE governing the transversely projected three gluon vertex was derived. To make contact, we consider the $L(q, r, p)$ of Eq. (5.3), and carry our the substitution $W_{\alpha \mu \nu}(q, r, p) \rightarrow$ $W_{\alpha \mu \nu}^{\mathrm{SDE}}(q, r, p)$, where

$$
W_{\alpha \mu \nu}^{\mathrm{SDE}}(q, r, p)=\bar{\Gamma}_{\alpha \mu \nu}^{(0)}(q, r, p),
$$

denoting the resulting expression by $L^{\mathrm{SDE}}(q, r, p)$.

Expanding $\Gamma^{\alpha \mu \nu}(q, r, p)$ in the basis of Eqs. (3.3) and (3.5) and substituting into Eq. (5.3), one may express $L^{\mathrm{SDE}}(q, r, p)$ in terms of the various $X_{i}$ and $Y_{i}$. Here, we do not report the general expression for $L^{\mathrm{SDE}}(q, r, p)$, but consider instead the following representative kinematic limits:

(i) Totally symmetric configuration: Fixing the momenta and the angle $\theta$ according to Eq. (4.10), one obtains

$$
\begin{aligned}
L^{\mathrm{SDE}}(Q)= & X_{1}(Q)-\frac{10}{11} Q^{2} X_{3}(Q)+\frac{5}{11} Q^{4} Y_{1}(Q) \\
& -\frac{4}{11} Q^{2} Y_{4}(Q) .
\end{aligned}
$$

(ii) Orthogonal-symmetric: In this configuration, the momenta $q$ and $r$ are orthogonal and have equal magnitudes, i.e., $\theta=\pi / 2$ and $q^{2}=r^{2}$, which also implies that $p^{2}=2 r^{2}$. In this case, the corresponding projection yields

$$
\begin{aligned}
L^{\mathrm{SDE}} & \left(r^{2}, r^{2}, \pi / 2\right) \\
= & \frac{1}{7}\left[X_{1}\left(r^{2}, r^{2}, \pi / 2\right)+6 X_{1}\left(2 r^{2}, r^{2}, 3 \pi / 4\right)\right. \\
& -r^{2} X_{3}\left(r^{2}, r^{2}, \pi / 2\right)-8 r^{2} X_{3}\left(2 r^{2}, r^{2}, 3 \pi / 4\right) \\
& +r^{4} Y_{1}\left(r^{2}, r^{2}, \pi / 2\right)+4 r^{4} Y_{1}\left(2 r^{2}, r^{2}, 3 \pi / 4\right) \\
& \left.-3 r^{2} Y_{4}\left(r^{2}, r^{2}, \pi / 2\right)\right] .
\end{aligned}
$$

(iii) Asymmetric limit: Fixing the momenta according to Eq. (4.12), we obtain

$L^{\mathrm{SDE}}(q)=X_{1}\left(q^{2}, q^{2}, \pi\right)-q^{2} X_{3}\left(q^{2}, q^{2}, \pi\right)$.

Note that the above kinematic configuration also corresponds to the so-called "orthogonal soft" limit, obtained in $[14,83] .{ }^{5}$ To establish their equivalence, first notice that Eq. (5.3), with the $W_{\alpha \mu \nu}^{\mathrm{SDE}}(q, r, p)$ given by Eq. (5.4), is symmetric under $p \leftrightarrow q$.

\footnotetext{
${ }^{5}$ The orthogonal soft configuration defined in [14] corresponds to the limit $q \rightarrow 0$ and $\theta=\pi / 2$.
} 

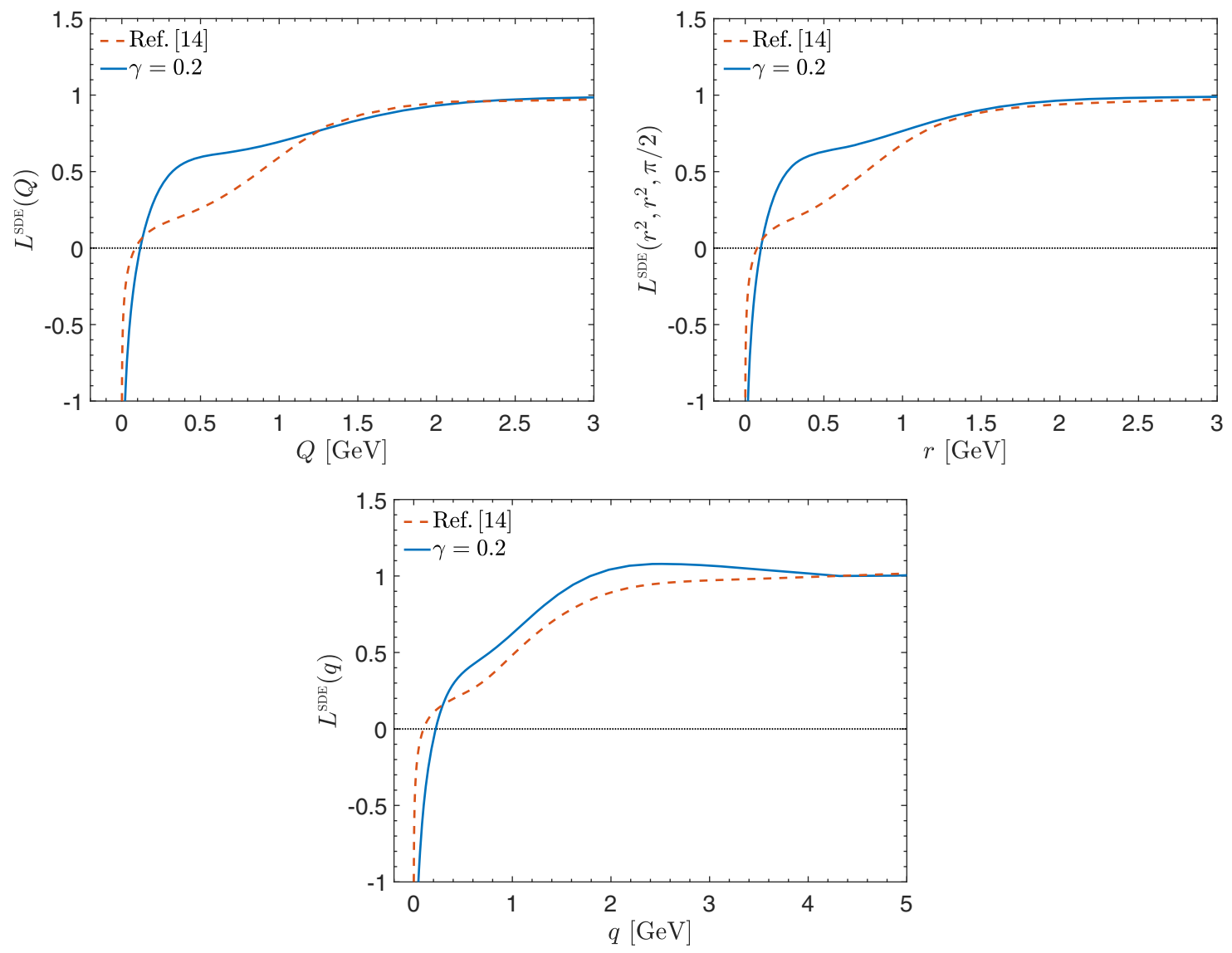

FIG. 15. Comparison of our results for $L^{\mathrm{SDE}}$ with those of [14,83] for three kinematics: (i) the totally symmetric $L^{\mathrm{SDE}}(Q)$ (top left panel), (ii) the orthogonal-symmetric $L^{\mathrm{SDE}}\left(r^{2}, r^{2}, \pi / 2\right)$ (top right panel), and (iii) asymmetric $L^{\mathrm{SDE}}(q)$ (bottom panel) configurations.

Therefore, the limits of vanishing $p$ or $q$ lead to the same result. In addition, when $q \rightarrow 0$, evidently $|q \| r| \cos \theta=0$, and any dependence on the angle $\theta$ is washed out. Thus, $L^{\mathrm{SDE}}\left(q^{2}, q^{2}, \pi\right)=$ $L^{\mathrm{SDE}}\left(0, r^{2}, \pi / 2\right)$.

An interesting property of the asymmetric configuration is the fact that $L^{\mathrm{SDE}}(q)$ depends only on the $X_{i}$, while the other two limits depend on both the $X_{i}$ and the $Y_{i}$. Since our approach does not allow the determination of $\Gamma_{\mathbf{T}}(q, r, p)$, in what follows we set $Y_{i}=0$ in Eqs. (5.5) and (5.6).

Evidently, the omission of the transverse form factors $Y_{i}$ in the evaluation of $L^{\mathrm{SDE}}(Q)$ and $L^{\mathrm{SDE}}\left(r^{2}, r^{2}, \pi / 2\right)$ introduces an error, whose size in the infrared is difficult to estimate without a concrete calculation. At this point we may only report the perturbative behavior of the terms omitted from Eq. (5.5), using the one-loop expressions for $Y_{1}(Q)$ and $Y_{4}(Q)$ given in Eq. (A3). In particular, one finds that the one-loop combination amounts only to a small constant, namely

$$
\frac{5}{11} Q^{4} Y_{1}^{(1)}(Q)-\frac{4}{11} Q^{2} Y_{4}^{(1)}(Q)=0.039 .
$$

Unfortunately, the perturbative calculation for the transverse terms omitted in Eq. (5.6) is more cumbersome, since it mixes the $Y_{i}$ in two kinematic limits.
In order to compare our results with the SDE calculations of [14], we first rescale the results appropriately, in order to ensure that they are renormalized at the same point. Specifically, we define a multiplicative renormalization constant $z_{3}$ for both sets of results, such that tree level value is restored at the symmetric point, i.e., $z_{3} L^{\mathrm{SDE}}(\mu)=1$; subsequently, we rescaled $L^{\mathrm{SDE}}\left(r^{2}, r^{2}, \pi / 2\right)$ and $L^{\mathrm{SDE}}(q)$ by the same factor.

In Fig. 15, we compare our results for $L^{\mathrm{SDE}}(Q)$ (left panel), $L^{\mathrm{SDE}}\left(r^{2}, r^{2}, \pi / 2\right)$ (right panel), and $L^{\mathrm{SDE}}(q)$ (bottom panel) with those obtained in $[14,83]$. The general profiles of the curves are qualitatively similar, in the three kinematic limits, although considerable differences are observed at intermediate momenta. Interestingly enough, the positions of the corresponding zero crossings practically coincide in all configurations.

\section{B. Comparison with the lattice}

Following the analysis presented in [25], we consider two particular cases of the $L(q, r, p)$ defined in Eq. (5.3).

First, for the symmetric configuration, we construct $L^{\mathrm{sym}}(Q)$ by setting $W_{\alpha \mu \nu}(q, r, p) \rightarrow W_{\alpha \mu \nu}^{\mathrm{sym}}(q, r, p)$, where 

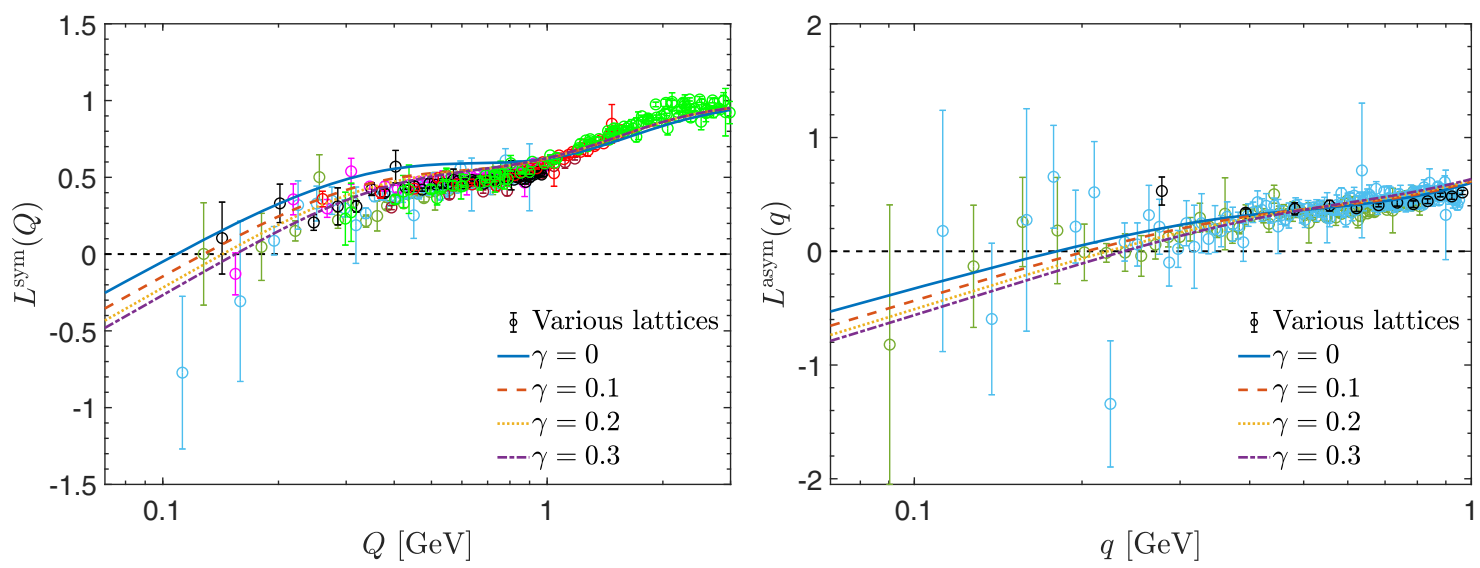

FIG. 16. Comparison of our results for the $L^{\mathrm{sym}}(Q)$ (left panel) given by Eq. (5.13) and for $L^{\text {asym }}(q)$ (right panel) of Eq. (5.12) with the lattice data [25] (circles). The curves were obtained by varying the exponent $\gamma$ entering into the definition of the gluon mass given by Eq. (4.3).

$W_{\alpha \mu \nu}^{\mathrm{sym}}(q, r, p)=\bar{\Gamma}_{\alpha \mu \nu}^{(0)}(q, r, p)+\frac{1}{2 r^{2}}(r-p)_{\alpha}(p-q)_{\mu}(q-r)_{\nu}$,

implementing subsequently the limit of Eq. (4.10).

Second, for the asymmetric configuration, we evaluate $L^{\mathrm{asym}}(q)$ by replacing $W_{\alpha \mu \nu}(q, r, p) \rightarrow W_{\alpha \mu \nu}^{\mathrm{asym}}(q, r, p)$, where

$$
W_{\alpha \mu \nu}^{\mathrm{asym}}(q, r, p)=2 q_{\alpha} P_{\mu \nu}(q),
$$

taking finally the limit of Eq. (4.12).

Expanding again $\Gamma_{\alpha \mu \nu}(q, r, p)$ in the basis of Eqs. (3.3) and (3.5), one finds that, in the symmetric configuration, Eq. (5.3) reduces to

$L^{\mathrm{sym}}(Q)=X_{1}(Q)-\frac{Q^{2}}{2} X_{3}(Q)+\frac{Q^{4}}{4} Y_{1}(Q)-\frac{Q^{2}}{2} Y_{4}(Q)$,

while for the asymmetric case,

$$
L^{\operatorname{asym}}(q)=X_{1}\left(q^{2}, q^{2}, \pi\right)-q^{2} X_{3}\left(q^{2}, q^{2}, \pi\right) .
$$

Thus, the combinations of form factors given in Eqs. (5.11) and (5.12) are precisely those considered in the lattice simulations of [25,28]. Notice that $L^{\mathrm{asym}}(q)$ given by Eq. (5.12) coincides with the projection $L^{\mathrm{SDE}}(q)$ of Eq. (5.7).

Since our approach furnishes no information on $\Gamma_{\mathbf{T}}(q, r, p)$, we will consider the approximate version of Eq. (5.11) where $Y_{i}=0$, as was done in the previous subsection. More specifically,

$$
L^{\mathrm{sym}}(Q)=X_{1}(Q)-\frac{Q^{2}}{2} X_{3}(Q),
$$

which will be used in the comparison with the lattice data. Let us simply mention that the one-loop evaluation of the omitted terms gives rise to a small numerical constant,

$$
\frac{Q^{4}}{4} Y_{1}^{(1)}(Q)-\frac{Q^{2}}{2} Y_{4}^{(1)}(Q)=0.08
$$

Note that, in order to perform a meaningful comparison, one must take into account the fact that the lattice results of [25] have been renormalized in a scheme which enforces independently that $L^{\mathrm{sym}}(\mu)=1$ and $L^{\text {asym }}(\mu)=1$ at the renormalization point $\mu=4.3 \mathrm{GeV}$. Instead, we have computed the $X_{i}$ in the Taylor scheme, for both kinematic limits [see discussion at the end of Sec. III]. To account for the difference introduced by the use of two distinct renormalization prescriptions, we rescale Eqs. (5.12) and (5.13) by a finite renormalization constant, to be denoted by $z_{3}$, i.e.,

$$
\begin{aligned}
& L^{\mathrm{sym}}(Q) \rightarrow z_{3}^{\mathrm{sym}} L^{\mathrm{sym}}(Q), \\
& L^{\operatorname{asym}}(q) \rightarrow z_{3}^{\mathrm{asym}} L^{\operatorname{asym}}(q) .
\end{aligned}
$$

The numerical values of $z_{3}^{\text {sym }}$ and $z_{3}^{\text {asym }}$ are determined by requiring that $L^{\mathrm{sym}}(\mu)$ and $L^{\mathrm{asym}}(\mu)$ reduce to tree level, respectively. As expected on general grounds [122], the discrepancy from unity is quite small; in particular, the choices of $z_{3}^{\text {sym }}=0.95$ and $z_{3}^{\text {asym }}=0.93$ restore $L^{\operatorname{sym}}(\mu)=$ 1 and $L^{\text {asym }}(\mu)=1$, respectively.

In Fig. 16 we compare the lattice data of [25] with our results for $L^{\mathrm{sym}}(q)$ (left panel) and $L^{\text {asym }}(q)$ (right panel), obtained for the different $J(q)$ shown in Fig. 4; evidently, in both cases the agreement is rather good. In fact, observe that, in the symmetric case, the lattice data display a change in the curvature (in the form of a "knee") around 
$Q=1 \mathrm{GeV}$. It is interesting to notice that, independently of the $J(q)$ employed, our results for $L^{\operatorname{sym}}(q)$ always exhibit this particular feature, which is clearly related to the abrupt bending observed at the level of the $X_{1}(Q)$ in the Fig. 13. Moreover, both the lattice data and our results present the characteristic zero crossing, at momenta to be denoted by $q_{0}^{\text {sym }}$ and $q_{0}^{\text {asym }}$, respectively, whose positions are located within the interval $[109,237] \mathrm{MeV}$ [see Table I]. Notice that, within our approach, the precise location of the crossing $q_{0}$ depends on the value of the parameter $\gamma$, which controls the running of $m^{2}\left(q^{2}\right)$ in Eq. (4.3).

It is interesting to compute the amount by which $q_{0}^{\text {sym }}$ and $q_{0}^{\text {asym }}$ get shifted with respect to $q_{0}^{\mathrm{J}}$, shown in Fig. 4; in Table I we collect these numbers, in order to facilitate a direct comparison. As one may see, for all values of $\gamma$ the crossing of $L^{\mathrm{sym}}(Q)$ happens at a momentum about $23 \%$ smaller than the value obtained from $J(q)$. In the case of the $L^{\text {asym }}(q)$, the change in the sign occurs at a momentum that is located $17 \%-28 \%$ more toward the ultraviolet with respect to $q_{0}^{\mathrm{J}}$.

\section{FURTHER CONSIDERATIONS AND CLARIFICATIONS}

In this section we comment on a number of subtleties related with some of the concepts introduced, and provide certain clarifications that we consider necessary.

(i) It should be evident that, while $\Gamma^{\alpha \mu \nu}$ has been expanded in the $\mathrm{BC}$ basis, mainly in order to make contact with the original $\mathrm{BC}$ construction, the term $V^{\alpha \mu \nu}$, given in Eqs. (2.8) and (2.12), is written in a
TABLE I. Comparison of the crossing positions $q_{0}^{\mathrm{J}}[J(q)]$, $q_{0}^{\text {sym }}\left[L^{\text {sym }}(Q)\right]$, and $q_{0}^{\text {asym }}\left[L^{\text {asym }}(q)\right]$.

\begin{tabular}{lccc}
\hline \hline$\gamma$ & $q_{0}^{\mathrm{J}}[$ in $\mathrm{MeV}]$ & $q_{0}^{\text {sym }}[$ in $\mathrm{MeV}]$ & $q_{0}^{\text {asym }}[$ in $\mathrm{MeV}]$ \\
\hline 0 & 140 & 109 & 180 \\
0.1 & 166 & 128 & 204 \\
0.2 & 187 & 143 & 221 \\
0.3 & 202 & 155 & 237 \\
\hline \hline
\end{tabular}

"naive" basis, whose elements, $n_{i}^{\alpha \mu \nu}$, are listed in Eq. (B2). From the transformation rules relating the form factors of both bases, given in Eqs. (B3) and (B4), it becomes clear that, in general, terms that are "longitudinally coupled," in the sense defined in the context of Eq. (2.8), when written in the $\mathrm{BC}$ basis formed by the tensors given in Eqs. (3.4) and (3.6), may have nonvanishing longitudinal and transverse components. ${ }^{6}$ For instance, to fix the ideas, let us consider the term $v^{\alpha \mu \nu}=q^{\alpha} r^{\mu} p^{\nu}$, which is one of the elements appearing in $V_{\alpha \mu \nu}(q, r, p)$, multiplied by $q^{-2} p^{-2} r^{-2}$; in the BC basis, it may be decomposed into longitudinal and transverse components, as shown in Eqs. (B6) and (B7). It is interesting to note the proliferation of terms needed for writing in the $\mathrm{BC}$ basis a term as simple as $v^{\alpha \mu \nu}$.

(ii) The previous exercise indicates that, when the entire $V_{\alpha \mu \nu}(q, r, p)$ of Eq. (2.8) is cast in the BC basis, namely

$$
V^{\alpha \mu \nu}(q, r, p)=\sum_{i=1}^{10} \mathcal{X}_{i}(q, r, p) \ell_{i}^{\alpha \mu \nu}(q, r, p)+\sum_{i=1}^{4} \mathcal{Y}_{i}(q, r, p) t_{i}^{\alpha \mu \nu}(q, r, p)
$$

it will contain both longitudinal and transverse components. To appreciate this point in a simplified context, let us consider the "Abelian" version of $V_{\alpha \mu \nu}(q, r, p)$, denoted by $\hat{V}_{\alpha \mu \nu}(q, r, p)$, obtained by setting $F=1$ and $H_{\nu \mu}=g_{\nu \mu}$ in Eq. (2.12), such that

$$
\begin{aligned}
& \hat{A}_{\mu \nu}(q, r, p)=\frac{1}{2}\left\{m^{2}\left(r^{2}\right) P_{\sigma \mu}(r)\left[g_{\nu}^{\sigma}+P_{\nu}^{\sigma}(p)\right]-m^{2}\left(p^{2}\right) P_{\sigma \nu}(p)\left[g_{\mu}^{\sigma}+P_{\mu}^{\sigma}(r)\right]\right\}, \\
& \hat{B}_{\alpha \nu}(q, r, p)=\frac{1}{2}\left\{m^{2}\left(p^{2}\right) P_{\sigma \nu}(p)\left[g_{\alpha}^{\sigma}+P_{\alpha}^{\sigma}(q)\right]-m^{2}\left(q^{2}\right) P_{\sigma \alpha}(q)\left[g_{\nu}^{\sigma}+P_{\nu}^{\sigma}(p)\right]\right\}, \\
& \hat{C}_{\alpha \mu}(q, r, p)=\frac{1}{2}\left\{m^{2}\left(q^{2}\right) P_{\sigma \alpha}(q)\left[g_{\mu}^{\sigma}+P_{\mu}^{\sigma}(r)\right]-m^{2}\left(r^{2}\right) P_{\sigma \mu}(r)\left[g_{\alpha}^{\sigma}+P_{\alpha}^{\sigma}(q)\right]\right\} .
\end{aligned}
$$

Then, expanding $\hat{V}_{\alpha \mu \nu}(q, r, p)$ in the $\mathrm{BC}$ basis, using the transformation formulas given in Appendix $\mathrm{B}$, we obtain

\footnotetext{
${ }^{6}$ Note that the elements $\ell_{2}^{\alpha \mu \nu}, \ell_{5}^{\alpha \mu \nu}$, and $\ell_{8}^{\alpha \mu \nu}$ of the BC basis are both "longitudinal," since they do not vanish when contracted by any of the external momenta, and "longitudinally coupled," because they satisfy Eq. (2.9).
} 


$$
\begin{aligned}
& \hat{\mathcal{X}}_{1}=-\frac{m^{2}\left(q^{2}\right)}{2 q^{2}}-\frac{m^{2}\left(r^{2}\right)}{2 r^{2}} ; \quad \hat{\mathcal{X}}_{2}=\frac{1}{2}\left(\frac{m^{2}\left(r^{2}\right)}{r^{2}}-\frac{m^{2}\left(q^{2}\right)}{q^{2}}\right) ; \quad \hat{\mathcal{X}}_{3}=\frac{q^{2} m^{2}\left(r^{2}\right)-r^{2} m^{2}\left(q^{2}\right)}{q^{2} r^{2}\left(q^{2}-r^{2}\right)} \\
& \hat{\mathcal{X}}_{4}=-\frac{m^{2}\left(p^{2}\right)}{2 p^{2}}-\frac{m^{2}\left(r^{2}\right)}{2 r^{2}} ; \quad \hat{\mathcal{X}}_{5}=\frac{1}{2}\left[\frac{m^{2}\left(p^{2}\right)}{p^{2}}-\frac{m^{2}\left(r^{2}\right)}{r^{2}}\right] ; \quad \hat{\mathcal{X}}_{6}=\frac{1}{p^{2}-r^{2}}\left[\frac{m^{2}\left(r^{2}\right)}{r^{2}}-\frac{m^{2}\left(p^{2}\right)}{p^{2}}\right] \\
& \hat{\mathcal{X}}_{7}=-\frac{m^{2}\left(p^{2}\right)}{2 p^{2}}-\frac{m^{2}\left(q^{2}\right)}{2 q^{2}} ; \quad \hat{\mathcal{X}}_{8}=\frac{1}{2}\left[\frac{m^{2}\left(q^{2}\right)}{q^{2}}-\frac{m^{2}\left(p^{2}\right)}{p^{2}}\right] ; \quad \hat{\mathcal{X}}_{9}=\frac{1}{p^{2}-q^{2}}\left[\frac{m^{2}\left(q^{2}\right)}{q^{2}}-\frac{m^{2}\left(p^{2}\right)}{p^{2}}\right] ; \\
& \hat{\mathcal{X}}_{10}=0 ; \\
& \hat{\mathcal{Y}}_{1}=\frac{q^{2}\left[-m^{2}\left(p^{2}\right)+m^{2}\left(q^{2}\right)-m^{2}\left(r^{2}\right)\right]+r^{2}\left[m^{2}\left(p^{2}\right)+m^{2}\left(q^{2}\right)-m^{2}\left(r^{2}\right)\right]}{p^{2} q^{2} r^{2}\left(q^{2}-r^{2}\right)} \\
& \hat{\mathcal{Y}}_{2}=\frac{\left(r^{2}-p^{2}\right)\left[m^{2}\left(q^{2}\right)-m^{2}\left(r^{2}\right)\right]+p^{2}\left[m^{2}\left(p^{2}\right)-2 m^{2}\left(r^{2}\right)\right]+r^{2} m^{2}\left(p^{2}\right)}{p^{2} q^{2} r^{2}\left(p^{2}-r^{2}\right)} \\
& \hat{\mathcal{Y}}_{3}=-\frac{m^{2}\left(r^{2}\right)\left(p^{2}-q^{2}\right)+\left[2\left(q^{2}+q \cdot r\right)+r^{2}\right]\left[m^{2}\left(q^{2}\right)-m^{2}\left(p^{2}\right)\right]}{p^{2} q^{2} r^{2}\left(p^{2}-q^{2}\right)} \\
& \hat{\mathcal{Y}}_{4}=\frac{(q \cdot r)\left[-m^{2}\left(p^{2}\right)+m^{2}\left(q^{2}\right)+m^{2}\left(r^{2}\right)\right]+q^{2} m^{2}\left(r^{2}\right)+r^{2} m^{2}\left(q^{2}\right)}{p^{2} q^{2} r^{2}}
\end{aligned}
$$

Evidently, since the $\hat{\mathcal{Y}}_{1}$ are nonvanishing, the transverse part of $\hat{V}_{\alpha \mu \nu}(q, r, p)$, and therefore that of the entire (Abelianized) vertex $\hat{\boldsymbol{\Gamma}}_{\alpha \mu \nu}(q, r, p)$, contains massless poles.

The generalization of the above construction to the full $V_{\alpha \mu \nu}(q, r, p)$ is straightforward but does not provide any further conceptual insights; the resulting expressions for the corresponding $\mathcal{X}_{i}$ and $\mathcal{Y}_{i}$ are quite lengthy, mainly due to the complicated "intertwining" between the mass terms and the $H_{\nu \mu}$ form factors, $A_{1}, A_{3}$, and $A_{4}$, and will not be reported here.

(iii) The main conclusion that one should draw from the above construction is that the expansion into the $\mathrm{BC}$ basis of the entire vertex $\boldsymbol{\Gamma}_{\alpha \mu \nu}(q, r, p)$, i.e., the sum of both $V_{\alpha \mu \nu}(q, r, p)$ and $\Gamma_{\alpha \mu \nu}(q, r, p)$, is of no practical usefulness, and may in fact be misleading. In particular, let us suppose for a moment that $\boldsymbol{\Gamma}_{\alpha \mu \nu}(q, r, p)$ [and not just $\Gamma_{\alpha \mu \nu}(q, r, p)$ as was done throughout this work] was indeed written in the BC basis. Then, the corresponding form factors, $\mathbb{X}_{i}$ and $\mathbb{V}_{i}$ would be simply given by

$$
\begin{aligned}
& \mathbb{X}_{i}=X_{i}+\mathcal{X}_{i}, \\
& \mathbb{Y}_{i}=Y_{i}+\mathcal{Y}_{i} ;
\end{aligned}
$$

of course, given the intrinsic limitations of the methodology employed in this work, we have no access to $Y_{i}$, but only to $\mathcal{Y}_{i}$. Evidently, due to the form of the $\mathcal{X}_{i}$ and $\mathcal{Y}_{i}$, all $\mathbb{X}_{i}$ and $\mathbb{Y}_{i}$ would be infested with massless poles; this, in turn, would be clearly reflected in the typical 3D plots of any individual $\mathbb{X}_{i}$ or $\mathbb{Y}_{i}$. However, any such representation would be physically disingenuous, because the pole terms from each $\mathbb{X}_{i}$ and $\mathbb{Y}_{i}$, when summed up, would eventually organize themselves into a "longitudinally coupled" contribution, namely none other than Eq. (2.8), and would cancel from physical amplitudes or lattice observables, such as the $L(q, p, r)$ of Eq. (5.3). Note, in particular, that, under these circumstances, it would be erroneous to consider, as part of an "approximation scheme," only the longitudinal part of the full $\boldsymbol{\Gamma}^{\alpha \mu \nu}$, to be denoted by $\boldsymbol{\Gamma}_{\mathbf{L}}^{\alpha \mu \nu}$, because one would then have, ${ }^{7}$

$$
P_{\alpha \alpha^{\prime}}(q) P_{\mu \mu^{\prime}}(r) P_{\nu \nu^{\prime}}(p) \boldsymbol{\Gamma}_{\mathbf{L}}^{\alpha \mu \nu}(q, r, p) \neq 0,
$$

and the final ("approximate") answer would be afflicted by the presence of spurious divergences.

The way the above problems have been resolved in the present work was simply by not expanding $V_{\alpha \mu \nu}(q, r, p)$ in the $\mathrm{BC}$ basis, which has been used exclusively for $\Gamma_{\alpha \mu \nu}(q, r, p)$, in order for the BCsolution to become directly applicable. Thus, the approximation employed amounts to setting $Y_{i}=0$, but keeping the entire $V_{\alpha \mu \nu}(q, r, p)$, or, in the language of the BC basis, both $\mathcal{X}_{i}$ and $\mathcal{Y}_{i}$ are present; and since Eq. (2.9) remains intact, what one determines and plots are the $X_{i}$, which contain no explicit massless poles.

(iv) The transverse form factors $Y_{i}$ of $\Gamma_{\alpha \mu \nu}(q, r, p)$, whose structures are undetermined by the present gauge-technique-based approach, may, in principle, contain divergent contributions, and, in particular, their own poles (simple, or of higher order). At

\footnotetext{
${ }^{7}$ This quantity was not introduced in the previous sections, precisely because of the subtleties associated with its nature.
} 
present, one may not exclude this possibility, and further independent studies, based on direct SDE approaches, may shed light on their structure. In such a search, the lattice results for $L^{\mathrm{sym}}(Q)$ provide an interesting constraint. Specifically, the nonobservability of pole divergences at the level of the $L^{\mathrm{sym}}(Q)$ requires that, in the limit $Q^{2} \rightarrow 0$,

$$
Q^{4} Y_{1}(Q)-2 Q^{2} Y_{4}(Q)=C,
$$

where $C$ is some arbitrary finite constant. Let us further assume, for the sake of argument, that the pole structure of $Y_{1}(Q)$ and $Y_{4}(Q)$ has the general form

$$
Y_{1}(Q)=\sum_{n=1}^{\infty} \frac{a_{n}}{Q^{2 n}}, \quad Y_{4}(Q)=\sum_{n=1}^{\infty} \frac{b_{n}}{Q^{2 n}}
$$

Then, Eq. (6.7) imposes the following constraints on the coefficients $b_{i}$ and $c_{i}$ :

$$
\begin{aligned}
a_{1} & =\text { undetermined, } \\
a_{2}-2 b_{1} & =C, \\
a_{n}-2 b_{n-1} & =0, \quad n=3,4, \ldots
\end{aligned}
$$

Evidently, the above constraints are trivially satisfied when all $a_{n}$ and $b_{n}$ vanish (in which case, $C=0$ ).

\section{ON THE "NAIVE" IMPLEMENTATION OF THE BC SOLUTION}

Finally, having provided a sufficient amount of background material, we may now revisit the central issue of the $\mathrm{BC}$ construction mentioned in the Introduction, namely the problems with using directly the term

$$
J_{\mathrm{BC}}(q)=\frac{\Delta^{-1}(q)}{q^{2}}
$$

as ingredient in the $\mathrm{BC}$ solution. In particular, let us assume that one were to ignore the presence and function of the term $V_{\alpha \mu \nu}(q, r, p)$, and suppose that the BC solution of Eq. (3.11) holds at the level of the $\mathbb{X}_{i}$, namely the full longitudinal form factors. As explained in the Introduction, due to the finiteness of the gluon propagator, $\Delta^{-1}(0)=m^{2}(0)$, the various $J_{\mathrm{BC}}(q)$ contain massless poles, which, through Eq. (3.11), will enter into the individual $\mathbb{X}_{i}$; and the combination of all such terms does not organize itself into a longitudinally coupled contribution.

The consequences of this scenario are rather striking. To appreciate this with one particular example, let us first reduce the algebraic complexity by turning off the ghost sector, and consider the "Abelianized" $\mathbb{X}_{i}$, given by Eq. (3.13), but now substitute $J(q) \rightarrow J_{\mathrm{BC}}(q)$, to obtain

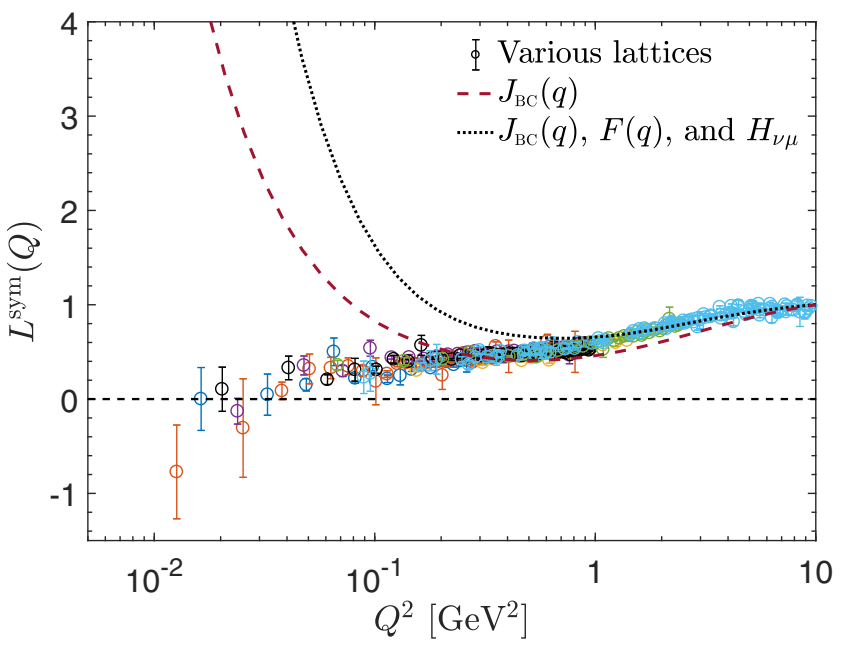

FIG. 17. Comparison of the results for $L_{\mathrm{BC}}^{\text {sym }}(Q)$ given by Eq. (7.2) (red dashed) with the lattice data of [25] (circles). $L^{\text {sym }}(Q)$ (black dotted curve) shows the impact of restoring the ghost sector. Evidently, the use of Eq. (7.1) produces a positive infrared divergence, which is incompatible with the lattice results.

(Euclidean space), $\hat{\mathbb{X}}_{1}(q, r, p)=\frac{1}{2}\left[J_{\mathrm{BC}}(r)+J_{\mathrm{BC}}(q)\right]$ and $\hat{\mathbb{X}}_{3}(q, r, p)=\frac{\left[J_{\mathrm{BC}}(q)-J_{\mathrm{BC}}(r)\right]}{r^{2}-q^{2}}$. Then, evaluate the lattice quantity $L^{\text {sym }}(Q)$, now to be denoted by $L_{\mathrm{BC}}^{\mathrm{sym}}(Q)$, by substituting $\hat{\mathbb{X}}_{1}$ and $\hat{\mathbb{X}}_{3}$ into Eq. (5.11), and setting (temporarily) $\hat{\mathbb{V}}_{1}(Q)=\hat{\mathbb{V}}_{4}(Q)=0$, to obtain

$$
\begin{aligned}
L_{\mathrm{BC}}^{\mathrm{sym}}(Q) & =J_{\mathrm{BC}}\left(Q^{2}\right)+\frac{Q^{2}}{2}\left(\frac{d J_{\mathrm{BC}}(Q)}{d Q^{2}}\right) \\
& =\frac{1}{2}\left[\frac{\Delta^{-1}(Q)}{Q^{2}}+\frac{d \Delta^{-1}(Q)}{d Q^{2}}\right] .
\end{aligned}
$$

In the limit $Q^{2} \rightarrow 0$, the first term is dominant, diverging as a simple pole,

$$
\lim _{Q^{2} \rightarrow 0} L_{\mathrm{BC}}^{\mathrm{sym}}(Q)=\frac{1}{2} \frac{m^{2}(0)}{Q^{2}}+\cdots
$$

where the ellipses denote subdominant terms (remember, in particular, that the derivative term diverges logarithmically). This pole term, in turn, is clearly visible when contrasting the $L_{\mathrm{BC}}^{\mathrm{sym}}(Q)$ with the lattice data, as shown in Fig. 17; thus, due to the huge discrepancy observed, the use of Eq. (7.1), at least under the assumptions leading to Eq. (7.3), is plainly discarded. Note also that the restoration of the ghost sector does not change the situation qualitatively; its inclusion simply increases the numerical value of the residue of the pole, making the onset of the divergence appear at higher values of $Q^{2}$, as seen in Fig. 17.

It is clear that the only way to circumvent this discrepancy and still use Eq. (7.1) is to relax the assumption that $\hat{\mathbb{V}}_{1}(Q)=\hat{\mathbb{\mho}}_{4}(Q)=0$; in fact, one ought to allow these 
latter form factors to have poles, which would precisely cancel the corresponding contribution in Eq. (7.3). Put in other words, in the absence of a concrete connection between the saturation of the gluon propagator and the vertex under consideration, the $\mathrm{BC}$ construction may not stand on its own, but requires the inclusion of transverse pieces with a necessarily nontrivial pole content. In particular, let us assume that, in complete analogy to Eq. (6.8), $\hat{\mathbb{\mho}}_{1}(Q)$ and $\hat{\mathbb{V}}_{4}(Q)$ are given by

$$
\hat{\mathbb{V}}_{1}(Q)=\sum_{n=1}^{\infty} \frac{\alpha_{n}}{Q^{2 n}}, \quad \hat{\mathbb{V}}_{4}(Q)=\sum_{n=1}^{\infty} \frac{\beta_{n}}{Q^{2 n}} .
$$

Then, to accomplish the nonobservability of pole contributions in $L_{\mathrm{BC}}^{\mathrm{sym}}(Q)$, the constraints of Eq. (6.9) must hold unchanged $\left(a_{n} \rightarrow \alpha_{n}, b_{n} \rightarrow \beta_{n}\right)$, with the very crucial exception of $n=3$, which must be now modified to

$$
\alpha_{3}-2 \beta_{2}=-2 m^{2}(0) ;
$$

evidently, the fulfillment of this last condition requires that at least one of the $\alpha_{3}$ and $\beta_{2}$ be nonvanishing.

Thus, in order for the "naive" BC construction to be compatible with the lattice results, the transverse part of the vertex must possess the minimal pole structure

$$
\hat{\mathbb{Y}}_{1}^{\min }(Q)=\frac{2 \beta_{2}-2 m^{2}(0)}{Q^{6}}, \quad \hat{\mathbb{V}}_{4}^{\min }(Q)=\frac{\beta_{2}}{Q^{4}} .
$$

To make the final connection, turn to the expressions for $\hat{\mathcal{Y}}_{1}$ and $\hat{\mathcal{Y}}_{4}$ given in (6.3), pass to Euclidean momenta, and compute them in the symmetric limit; it is fairly straightforward to establish that

$$
\hat{\mathcal{Y}}_{1}(Q)=\frac{m^{2}(Q)}{Q^{6}}-\frac{2}{Q^{4}} \frac{d m^{2}(Q)}{d Q^{2}}, \quad \hat{\mathcal{Y}}_{4}(Q)=\frac{3 m^{2}(Q)}{2} .
$$

If at this point one were to identify $\hat{\mathcal{Y}}_{1}(Q)$ and $\hat{\mathcal{Y}}_{4}(Q)$ with $\hat{\mathbb{Y}}_{1}(Q)$ and $\hat{\mathbb{Y}}_{4}(Q)$, respectively (which is tantamount to using Eq. (6.5) with $Y_{i}=0$ ), in the limit of $Q^{2} \rightarrow 0$ one would have

$$
\alpha_{3}=m^{2}(0), \quad \beta_{2}=\frac{3}{2} m^{2}(0),
$$

which satisfy precisely the no-pole condition of Eq. (7.5). ${ }^{8}$ Thus, the pole structure contained in the $\hat{\mathcal{Y}}_{1}$ and $\hat{\mathcal{Y}}_{4}(Q)$ is

\footnotetext{
${ }^{8}$ Since the first derivative of $m^{2}(Q)$ is finite at the origin [40,87], the remaining nonvanishing coefficient $\alpha_{2}=$ $-\left[\frac{2 d m^{2}(Q)}{d Q^{2}}\right]_{Q^{2}=0}$ fixes simply the value of $C$ through the second relation of Eq. (6.9).
}

identical to the minimal pole structure of Eq. (7.6), required for the compatibility with the lattice data.

We end this discussion with a final comparison between the "naive" $\mathrm{BC}$ version, described in this section, and the one presented in the main part of this work. Evidently, the "naive" implementation of the BC construction in "isolation" is physically incomplete, because it requires the $a$ posteriori inclusion of very precise transverse contributions. In particular, in the absence of lattice results, one would have no guiding principle on how to construct these terms, except through the imposition of the additional requirement that the combination $P_{\alpha \alpha^{\prime}}(q) P_{\mu \mu^{\prime}}(r) P_{\nu \nu^{\prime}}(p) \boldsymbol{\Gamma}^{\alpha \mu \nu}(q, r, p)$ be finite, which would lead essentially to the results of this section. Note, however, that this last requirement alone, although essentially correct, establishes no deeper connection with the dynamics of the two-point sector of the theory. Instead, the construction followed in this work adheres to the theoretical principles that have been spelled out in a series of articles, being intimately linked with the intricate dynamics taking place at the level of the gluon propagator, and, in particular, with the mass generating mechanism employed. In this way, the results turn out to be naturally compatible with the lattice, and no a-posteriori adjustments are required.

\section{CONCLUSIONS}

In the present work we have carried out the nonperturbative derivation of the longitudinal part of the three-gluon vertex, $\boldsymbol{\Gamma}_{\alpha \mu \nu}(q, r, p)$, from the set of STIs that it satisfies, given in Eq. (2.4). The procedure followed is a variant of the well-known BC construction [54], where certain key adjustments have been implemented in order to account for the fact that the gluon propagator appearing in the problem is infrared finite. In particular, in the context of the PT-BFM framework [34], the origin of the gluonic mass scale, $m^{2}(q)$, is attributed to the activation of the Schwinger mechanism by the longitudinally coupled massless poles, which constitute a purely nonperturbative component of the full vertex $\boldsymbol{\Gamma}_{\alpha \mu \nu}(q, r, p)$, denoted by $V_{\alpha \mu \nu}(q, r, p)$ [see Eq. (2.8)]. The inextricable link between $V_{\alpha \mu \nu}(q, r, p)$ and $m^{2}(q)$ leads to the partial STI given in Eq. (2.11) $[36,37,40,41,87]$, while the remainder of the vertex, denoted by $\Gamma_{\alpha \mu \nu}(q, r, p)$, satisfies the STI given in Eq. (2.10), which involves the kinetic term $J(q)$, appearing in the decomposition of Eq. (2.6). Given that $V_{\alpha \mu \nu}(q, r, p)$ is completely fixed by the STI of Eq. (2.8) and the condition of Eq. (2.9) [see Eq. (2.12)], the remaining task boiled down to the application of the $\mathrm{BC}$ solution at the level of the $\Gamma_{\alpha \mu \nu}(q, r, p)$, whose longitudinal form factors, $X_{i}$, may be thus obtained from the STI in Eq. (2.10) and its permutations.

The main ingredient entering in the $\mathrm{BC}$ solution is the function $J(q)$, whose form is determined indirectly, through the subtraction of $m^{2}(q)$ from $\Delta^{-1}(q)$. The most prominent feature of $J(q)$ is the zero crossing, whose origin 


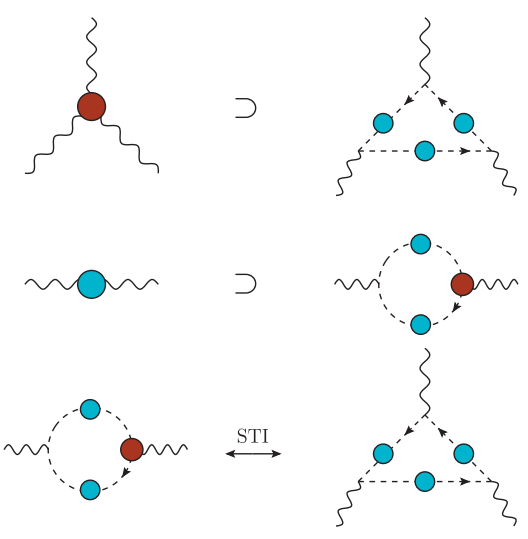

(a)

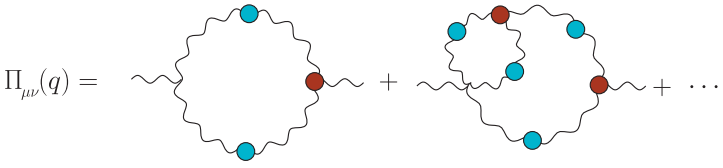

(b)

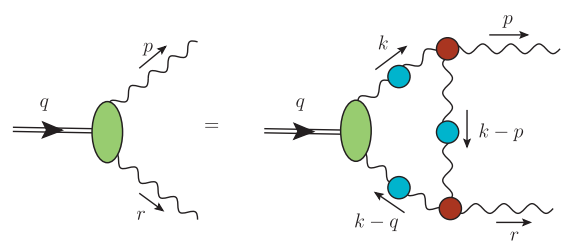

(c)

FIG. 18. Panel (a): The SDE diagram of the three gluon vertex responsible for the suppression (first row), the one in the gaugetechnique approach (second row), and their schematic connection implemented by the STI (third row). Panel (b): The gluon self-energy contributions containing the three gluon vertex. Panel (c): The homogeneous BSE which describes the formation of the (colored) massless excitations contained in $V_{\alpha \mu \nu}$, or one of the contributions entering in the glueball BSEs.

may be traced back to the "unprotected" logarithms contained in it. Note that the precise form of $J(q)$ is affected by the approximations implemented at the level of the dynamical equation that determines $m^{2}(q)$, and in particular the value of the coefficient $\gamma$ in the fit of Eq. (4.3). This fact, in turn, introduces minor uncertainties in the results for the form factors, such as the location of the corresponding zero crossings displayed by the $X_{i}$, but does not alter the main qualitative and quantitative aspects of the answer.

The most prominent feature of the longitudinal form factors is their distinct suppression at energies below $1 \mathrm{GeV}$. This property is clearly visible both in the 3D plots of Fig. 7 and Fig. 12, where the size of two form factors becomes inferior to unity (their tree-level value) for intermediate and infrared momenta. This same trait is also captured in the 2D plots, corresponding to the two special configurations studied [left panels of Fig. 13 and Fig. 14]. It is important to emphasize that, in addition to lattice simulations, this suppression has also been observed in the studies carried out using different approaches, such as the direct SDE-based derivations of [14,83], shown in Fig. 15.

It is interesting to comment on the origin of the suppression (and the zero-crossing) in the gauge-technique (BC solution) and the SDE analyses. Evidently, in both approaches the resulting suppression is the outcome of the "competition" between the infrared finite contributions originating from diagrams containing "massive" gluons and the infrared divergent logarithms stemming from diagrams containing massless ghosts. As the momenta become smaller, the "unprotected" logarithms take over, causing the overall suppression, which culminates with a negative logarithmic divergence at the origin. In the case of the SDE analysis, where the corresponding integral equation for $\Gamma_{\alpha \mu \nu}(q, r, p)$ is considered directly, the diagram responsible for the suppression is the triangle ghost graph [Fig. 18, first row of panel (a)]. Instead, in the case of the gauge-technique, where the form factors of $\Gamma_{\alpha \mu \nu}(q, r, p)$ are built out of the quantities appearing in the corresponding STIs, the ingredient causing the suppression and the zero crossing is the ghost loop that appears in the diagrammatic expansion of the gluon propagator [Fig. 18, second row of panel (a)]. This particular graph furnishes unprotected logarithms, which eventually find their way into the structure of the function $J(q)$. Evidently, the STIs relate these two graphs, as shown schematically in Fig. 18, third row of panel (a).

The 3D results obtained for the $X_{i}$ may be employed in a variety of situations where the three-gluon vertex is expected to play a significant role, and especially in circumstances where integrations over the entire range of momenta are required. In what follows we will mention a few notable cases that belong to this general category.

The three-gluon vertex is instrumental for the SDE that governs the momentum evolution of the gluon propagator, entering in the diagrams shown in the panel (b) of Fig. 18. After the implementation of the decompositions given in Eqs. (2.6) and (2.7), the original SDE furnishes two integral equations [36,86], which determine the quantities $J(q)$ and $m^{2}(q)$. The use of the 3D data obtained here for the $X_{i}$, instead of approximate Ansätze, is expected to provide a tighter control on the behavior of these two quantities.

As has been shown in [37,87], the formation of the (colored) massless excitations contained in $V_{\alpha \mu \nu}(q, r, p)$ hinges on the nonvanishing of the vertex function $\mathcal{B}_{\mu \nu}$, introduced in Fig. 2. This possibility, in turn, is determined by the homogeneous BSE shown in panel (c) of Fig. 18, where the "green ellipse" represents $\mathcal{B}_{\mu \nu}$. Evidently, the kernel of this BSE, and hence the type of solutions obtained, depend crucially on the details of the product $\boldsymbol{\Gamma} \boldsymbol{\Gamma}$; in the early treatments cited above, this product was simply approximated by its tree-level value, i.e., $\boldsymbol{\Gamma} \boldsymbol{\Gamma} \rightarrow \Gamma^{(0)} \Gamma^{(0)}$, and nontrivial solutions were found that corroborate the proposed mass generating mechanism. In fact, the solutions 
obtained are intimately related with the first derivative of the running gluon mass, $d m^{2}\left(q^{2}\right) / d q^{2}$ [87], from which $m^{2}\left(q^{2}\right)$ may be reconstructed [40]. The detailed knowledge of the $X_{i}$ allows for a more sophisticated treatment of this problem, achieving a higher degree of self-consistency between all the ingredients involved.

The treatment of systems of BSEs is of central importance in the studies dedicated to the formation of glueballs. The particular BSE shown in panel (c) of Fig. 18 is present in all such analyses. ${ }^{9}$ Previous studies indicate that in order to obtain masses compatible with lattice simulations, the total integrated strength of the kernel must undergo a considerable suppression [43,46]. A need for an analogous attenuation has been also observed in the recent study of hybrid mesons [47]; the required suppression has been implemented by resorting to a simplified Ansatz for the form factors associated with the tensors comprising $\Gamma_{\alpha \mu \nu}^{(0)}(q, r, p)$. Evidently, the results obtained here offer the possibility of refining future studies in this direction, furnishing ingredients that, despite the approximations implemented in deriving them, can trace their origins to the fundamental underlying theory.

An important limitation of the method employed in this work is that the structure of the transverse form factors $Y_{i}$ of the vertex $\Gamma_{\alpha \mu \nu}(q, r, p)$ [see Eqs. (3.5) and (6.5)] remains completely undetermined. Of course, this particular drawback is typical to all gauge-technique based approaches, even though, in some cases, such as the electron-photon or the quark-gluon vertices, partial information on these form factors may be extracted from the so-called "transverse" Ward identities [123-128]. Note that the $Y_{i}$ may be numerically relevant in some of the problems mentioned earlier. Moreover, their inclusion is important in situations where $\Gamma_{\alpha \mu \nu}(q, r, p)$ forms part of an integral equation that must be multiplicatively renormalized; a particularly relevant example of such a case is again the SDE appearing in the panel (b) of Fig. 18. A systematic way for obtaining approximate expressions for these components could be developed at the level of the $\mathrm{SDE}$ satisfied by $\Gamma_{\alpha \mu \nu}(q, r, p)$, where the tensorial structures associated with the $Y_{i}$ will have to be appropriately projected out of the corresponding integral equation. Calculations in that direction are already underway, and we hope to report on the results in the near future.

\section{ACKNOWLEDGMENTS}

The research of J. P. is supported by the Spanish Ministry of Economy and Competitiveness (MINECO) under Grants No. FPA2017-84543-P and No. SEV-2014-0398, and Generalitat Valenciana under Grant No. Prometeo II/

\footnotetext{
${ }^{9}$ The "green ellipse" in Fig. 18 represents now the corresponding glueball amplitudes. Note also that the color structure of the problem is different than that of the massless colored excitations mentioned above.
}

2014/066. The work of A. C.A and M. N. F. are supported by the Brazilian National Council for Scientific and Technological Development $(\mathrm{CNPq})$ under the Grants No. 305815/2015 and No. 142226/2016-5, respectively. A. C. A and C. T. F. also acknowledge the financial support from São Paulo Research Foundation (FAPESP) through the Projects No. 2017/07595-0, No. 2017/05685-2, No. 2016/ 11894-0, and No. 2018/09684-3. This study was financed in part by the Coordenação de Aperfeiçoamento de Pessoal de Nível Superior-Brasil (CAPES)—Finance Code 001 (M. N. F.). This research was performed using the Feynman Cluster of the John David Rogers Computation Center (CCJDR) in the Institute of Physics "Gleb Wataghin," University of Campinas.

\section{APPENDIX A: ONE-LOOP RESULTS}

In this Appendix we present the one-loop results for form factors in the (i) totally symmetric, (ii) asymmetric, and (iii) the general orthogonal configurations. All of them were obtained by the direct evaluation of the one-loop diagrams contributing to the three-gluon vertex. The relevant expressions are obtained from [88] (Landau gauge), and are renormalized in the Taylor scheme; this means that the corresponding $A_{i}$ are not renormalized at $Q^{2}=\mu^{2}$, but rather at the "soft-ghost" configuration, as discussed at the end of Sec. III.

In particular, $Z_{3}$ will be obtained directly from Eq. (3.15), by setting

$$
\begin{aligned}
& Z_{A}=1+\frac{C_{\mathrm{A}} \alpha_{s}}{144 \pi}\left[78\left\{\frac{2}{\epsilon}-\gamma_{E}+\ln (4 \pi)\right\}+97\right], \\
& Z_{c}=1+\frac{C_{\mathrm{A}} \alpha_{s}}{16 \pi}\left[3\left\{\frac{2}{\epsilon}-\gamma_{E}+\ln (4 \pi)\right\}+4\right], \\
& Z_{1}=1,
\end{aligned}
$$

where $\gamma_{E}$ denotes the Euler-Mascheroni constant, and $\epsilon=4-d$, with $d$ being the dimension of spacetime in dimensional regularization.

Substituting the results of Eqs. (A1) into the STI for the renormalization constants given by (3.15), one finds

$$
Z_{3}=1+\frac{C_{\mathrm{A}} \alpha_{s}}{144 \pi}\left[51\left\{\frac{2}{\epsilon}-\gamma_{E}+\ln (4 \pi)\right\}+61\right] .
$$

Then, one may obtain ultraviolet finite (cutoff-independent) one-loop results for the three-gluon vertex by performing the renormalization as described above. In what follows we will implement this procedure to obtain the corresponding results for the symmetric and asymmetric configurations.

(1) Symmetric configuration The kinematics of this configuration is defined in Eq. (4.10). Then, at one-loop we have ${ }^{10}$

\footnotetext{
${ }^{10}$ The corresponding two-loop calculation in the $\overline{\mathrm{MS}}$ was presented in [129].
} 
$X_{1}^{(1)}(Q)=1+\frac{C_{\mathrm{A}} \alpha_{s}}{48 \pi}\left[17 \ln \left(\frac{Q^{2}}{\mu^{2}}\right)+3-3 \mathrm{I}\right]$,

$X_{2}^{(1)}(Q)=0$,

$X_{3}^{(1)}(Q)=-\frac{C_{\mathrm{A}} \alpha_{s}}{48 \pi Q^{2}}(38-7 \mathrm{I}), \quad X_{10}^{(1)}(Q)=0$,

$Y_{1}^{(1)}(Q)=-\frac{C_{\mathrm{A}} \alpha_{s}}{432 \pi Q^{4}}(587-193 \mathrm{I})$,

$Y_{4}^{(1)}(Q)=-\frac{C_{\mathrm{A}} \alpha_{s}}{864 \pi Q^{2}}(365+179 \mathrm{I})$,

with I defined as [122]

$$
\mathrm{I}=\frac{1}{3}\left[\psi_{1}\left(\frac{1}{3}\right)-\psi_{1}\left(\frac{2}{3}\right)\right]=2.34391
$$

where $\psi_{1}(z)$ is the trigamma function, related to $\Gamma(z)$ by

$$
\psi_{1}(z)=\frac{d^{2}}{d z^{2}} \ln [\Gamma(z)]
$$

The form factors $X_{2}$ and $X_{10}$ vanish in the symmetric configuration since they are antisymmetric under the exchange of at least two arguments [see Eq. (3.7)].

(2) Asymmetric configuration

In the asymmetric configuration, defined in Eq. (4.12), the tensor structure of the three-gluon vertex reduces to [88]

$$
\begin{aligned}
\Gamma_{\alpha \mu \nu}(q,-q, 0)= & 2 g_{\alpha \mu} q_{\nu}\left[X_{1}(q,-q, 0)-q^{2} X_{3}(q,-q, 0)\right]-2 q_{\alpha} q_{\mu} q_{\nu} X_{3}(q,-q, 0) \\
& -\left(q_{\alpha} g_{\mu \nu}+q_{\mu} g_{\alpha \nu}\right)\left[X_{1}(0, q,-q)-X_{2}(0, q,-q)\right] .
\end{aligned}
$$

Then, the corresponding form factors at one-loop read

$$
\begin{aligned}
X_{1}^{(1)}(q,-q, 0) & =1+\frac{17 C_{\mathrm{A}} \alpha_{s}}{48 \pi} \ln \left(\frac{q^{2}}{\mu^{2}}\right), \\
X_{3}^{(1)}(q,-q, 0) & =-\frac{37 C_{\mathrm{A}} \alpha_{s}}{96 \pi q^{2}}, \\
X_{1}^{(1)}(0, q,-q)-X_{2}^{(1)}(0, q,-q) & =X_{1}^{(1)}(q,-q, 0) .
\end{aligned}
$$

Notice that we cannot disentangle $X_{1}^{(1)}(0, q,-q)$ and $X_{2}^{(1)}(0, q,-q)$.

One should also note that, due to our choice of the Taylor renormalization prescription, $X_{1}$ reduces to its tree-level value for $q=\mu$ in the asymmetric configuration, $X_{1}^{(1)}(\mu,-\mu, 0)=1$, instead of satisfying this condition at the symmetric point.

(3) General orthogonal configuration

In this configuration, the momenta $q^{2}$ and $r^{2}$ are independent, but the angle $\theta$ is fixed at $\theta=\pi / 2$; therefore, one has $p^{2}=q^{2}+r^{2}$. In this case we have determined only $X_{1}$, which is given by

$$
\begin{aligned}
X_{1}\left(q^{2}, r^{2}, \pi / 2\right)= & 1+\frac{C_{\mathrm{A}} \alpha_{s}}{768 \pi q^{2} r^{2}}\left\{2\left(9 q^{4}+128 q^{2} r^{2}+3 r^{4}\right) \ln \left(\frac{q^{2}}{\mu^{2}}\right)-12\left(q^{4}-8 q^{2} r^{2}+r^{4}\right)\right. \\
& +2\left(3 q^{4}+128 q^{2} r^{2}+9 r^{4}\right) \ln \left(\frac{r^{2}}{\mu^{2}}\right)-24\left(q^{4}+10 q^{2} r^{2}+r^{4}\right) \ln \left(\frac{q^{2}+r^{2}}{\mu^{2}}\right) \\
& \left.-3 i \frac{\left(q^{2}+r^{2}\right)}{q r}\left(q^{2}-r^{2}\right)^{2}\left[\operatorname{Li}_{2}(-z)-\mathrm{Li}_{2}(z)+\mathrm{Li}_{2}\left(z^{-1}\right)-\mathrm{Li}_{2}\left(-z^{-1}\right)\right]\right\},
\end{aligned}
$$

with $q=|q|, r=|r|, z=(q-i r) /(q+i r)$, and

$$
\mathrm{Li}_{2}(z)=-\int_{0}^{z} \frac{\ln (1-t)}{t} d t
$$

is the dilogarithm (or Spence function). Note that the above expression is symmetric under $q \leftrightarrow r$. 
(4) Orthogonal symmetric configuration

This is a particular limit of Eq. (A8) where $q^{2}=r^{2}$. We obtain

$$
\begin{aligned}
& X_{1}\left(q^{2}, q^{2}, \pi / 2\right) \\
& =1+\frac{C_{\mathrm{A}} \alpha_{s}}{96 \pi}\left[34 \ln \left(\frac{q^{2}}{\mu^{2}}\right)-36 \ln (2)+9\right] .
\end{aligned}
$$

\section{APPENDIX B: THE BC AND NAIVE BASES}

Let us consider an arbitrary tensor with three Lorentz indices $(\alpha, \mu, \nu)$ and three momenta $(q, r, p)$, to be denoted by $S^{\alpha \mu \nu}(q, r, p)$. We expand $S^{\alpha \mu \nu}(q, r, p)$ in two different bases, the "naive" and the $\mathrm{BC}$ basis,

$$
\begin{aligned}
S^{\alpha \mu \nu}(q, r, p) & =\sum_{i=1}^{14} N_{i}(q, r, p) n_{i}^{\alpha \mu \nu}, \\
& =\sum_{i=1}^{10} L_{i}(q, r, p) \ell_{i}^{\alpha \mu \nu}+\sum_{i=1}^{4} T_{i}(q, r, p) t_{i}^{\alpha \mu \nu},
\end{aligned}
$$

where the $\mathrm{BC}$ elements $\ell_{i}^{\alpha \mu \nu}$ and $t_{i}^{\alpha \mu \nu}$ are given in Eqs. (3.4) and (3.6), and we define the elements of the naive basis to be

$$
\begin{aligned}
& n_{1}^{\alpha \mu \nu}=q^{\alpha} g^{\mu \nu} ; \quad n_{2}^{\alpha \mu \nu}=q^{\alpha} q^{\mu} q^{\nu} ; \quad n_{3}^{\alpha \mu \nu}=q^{\alpha} q^{\mu} r^{\nu} ; \quad n_{4}^{\alpha \mu \nu}=q^{\alpha} r^{\mu} q^{\nu} ; \quad n_{5}^{\alpha \mu \nu}=q^{\alpha} r^{\mu} r^{\nu}, \\
& n_{6}^{\alpha \mu \nu}=r^{\alpha} g^{\mu \nu} ; \quad n_{7}^{\alpha \mu \nu}=r^{\alpha} q^{\mu} q^{\nu} ; \quad n_{8}^{\alpha \mu \nu}=r^{\alpha} q^{\mu} r^{\nu} ; \quad n_{9}^{\alpha \mu \nu}=r^{\alpha} r^{\mu} q^{\nu} ; \quad n_{10}^{\alpha \mu \nu}=r^{\alpha} r^{\mu} r^{\nu}, \\
& n_{11}^{\alpha \mu \nu}=q^{\mu} g^{\nu \alpha} ; \quad n_{12}^{\alpha \mu \nu}=q^{\nu} g^{\mu \alpha} ; \quad n_{13}^{\alpha \mu \nu}=r^{\mu} g^{\nu \alpha} ; \quad n_{14}^{\alpha \mu \nu}=r^{\nu} g^{\mu \alpha} \text {. }
\end{aligned}
$$

The form factors $N_{i}$ can be written in terms of the form factors $L_{i}$ and $T_{i}$ as

$$
\begin{aligned}
N_{1} & =L_{4}-L_{5}-(p \cdot r) L_{6}+(p \cdot r)(q \cdot r) T_{2}-(q \cdot r) T_{4}, \\
N_{2} & =2 L_{9}+r^{2} T_{3}, \\
N_{3} & =-L_{6}-L_{10}+(q \cdot r) T_{2}+T_{4}, \\
N_{4} & =L_{9}-(q \cdot r) T_{3}, \\
N_{5} & =-L_{6}+(q \cdot r) T_{2}, \\
N_{6} & =2 L_{4}-2(p \cdot r) L_{6}-q^{2}(p \cdot r) T_{2}+q^{2} T_{4}, \\
N_{7} & =L_{3}+2 L_{9}-L_{10}-(p \cdot r) T_{1}+r^{2} T_{3}-T_{4}, \\
N_{8} & =-L_{3}-2 L_{6}-L_{10}+(p \cdot q) T_{1}-q^{2} T_{2}+T_{4}, \\
N_{9} & =L_{9}-L_{10}-(q \cdot r) T_{3}-T_{4}, \\
N_{10} & =-2 L_{6}-q^{2} T_{2}, \\
N_{11} & =-2 L_{7}+2(p \cdot q) L_{9}+r^{2}(p \cdot q) T_{3}-r^{2} T_{4}, \\
N_{12} & =L_{1}+L_{2}-(q \cdot r) L_{3}+(p \cdot r)(q \cdot r) T_{1}-(p \cdot r) T_{4}, \\
N_{13} & =-L_{7}-L_{8}+(p \cdot q) L_{9}-(p \cdot q)(q \cdot r) T_{3}+(q \cdot r) T_{4}, \\
N_{14} & =-L_{1}+L_{2}+(q \cdot r) L_{3}-(p \cdot q)(q \cdot r) T_{1}+(p \cdot q) T_{4},
\end{aligned}
$$

where we have omitted the momenta dependence of the form factors. 
On the other hand, the change of basis can be inverted in order to obtain $L_{i}$ and $T_{i}$ in terms of $N_{i}$,

$$
\begin{aligned}
& L_{1}=\frac{1}{4}\left\{2\left[(q \cdot r)\left(N_{10}-N_{2}+N_{3}+N_{4}-N_{5}+N_{7}-N_{8}-N_{9}\right)+N_{12}-N_{14}\right]\right. \\
& \left.+(p \cdot q)\left(N_{3}+N_{4}-N_{5}-N_{9}\right)+(p \cdot r)\left(N_{3}+N_{4}-N_{5}-N_{9}\right)\right\}, \\
& L_{2}=\frac{1}{4}\left\{2\left[(q \cdot r)\left(-N_{10}-N_{2}-N_{3}+N_{4}+N_{5}+N_{7}+N_{8}-N_{9}\right)+N_{12}+N_{14}\right]\right. \\
& \left.+(p \cdot q)\left(-N_{3}-N_{4}+N_{5}+N_{9}\right)+(p \cdot r)\left(N_{3}+N_{4}-N_{5}-N_{9}\right)\right\} \\
& L_{3}=\frac{(p \cdot r)\left(-N_{10}-N_{3}+N_{5}+N_{8}\right)+(p \cdot q)\left(-N_{2}+N_{4}+N_{7}-N_{9}\right)}{(p \cdot q)-(p \cdot r)} \text {, } \\
& L_{4}=\frac{1}{4}\left\{-2(p \cdot r) N_{10}+q^{2}\left(-N_{3}-N_{4}+N_{5}+N_{9}\right)+2 N_{6}\right\} \text {, } \\
& L_{5}=\frac{1}{4}\left\{2\left[-2 N_{1}+(q \cdot r)\left(-N_{3}-N_{4}+N_{5}+N_{9}\right)+N_{6}\right]-2(p \cdot r)\left(N_{10}-2 N_{5}\right)+q^{2}\left(-N_{3}-N_{4}+N_{5}+N_{9}\right)\right\} \text {, } \\
& L_{6}=-\frac{(q \cdot r) N_{10}+q^{2} N_{5}}{q^{2}+2(q \cdot r)} \\
& L_{7}=\frac{1}{4}\left\{-2 N_{11}+2(p \cdot q) N_{2}+r^{2}\left(-N_{3}-N_{4}+N_{5}+N_{9}\right)\right\} \\
& L_{8}=\frac{1}{4}\left\{2 N_{11}-4 N_{13}-2(p \cdot q)\left(N_{2}-2 N_{4}\right)+2(q \cdot r)\left(N_{3}+N_{4}-N_{5}-N_{9}\right)+r^{2}\left(N_{3}+N_{4}-N_{5}-N_{9}\right)\right\} \text {, } \\
& L_{9}=\frac{(q \cdot r) N_{2}+r^{2} N_{4}}{2(q \cdot r)+r^{2}} \\
& L_{10}=\frac{1}{2}\left(-N_{3}+N_{4}+N_{5}-N_{9}\right) \text {, } \\
& T_{1}=\frac{-N_{2}-N_{3}+N_{4}+N_{5}+N_{7}+N_{8}-N_{9}-N_{10}}{(p \cdot q)-(p \cdot r)}, \\
& T_{2}=\frac{2 N_{5}-N_{10}}{q^{2}+2(q \cdot r)} \text {, } \\
& T_{3}=\frac{N_{2}-2 N_{4}}{2(q \cdot r)+r^{2}} \text {, } \\
& T_{4}=\frac{1}{2}\left(N_{3}+N_{4}-N_{5}-N_{9}\right) \text {. }
\end{aligned}
$$

As a concrete example, consider the vector $v^{\alpha \mu \nu} \equiv q^{\alpha} r^{\mu} p^{\nu}$, which can be written in the naive basis as

$$
v^{\alpha \mu \nu}=-n_{4}^{\alpha \mu \nu}-n_{5}^{\alpha \mu \nu}
$$

i.e., $\quad N_{4}=N_{5}=-1$, while all the other form factors vanish. Using the transformation rules of Eq. (B4) (and $q+p+r=0$ ), we can write this vector in the $\mathrm{BC}$ basis as

$$
v^{\alpha \mu \nu}=v_{\mathbf{L}}^{\alpha \mu \nu}+v_{\mathbf{T}}^{\alpha \mu \nu}
$$

with

$$
\begin{aligned}
& v_{\mathbf{L}}^{\alpha \mu \nu}=-(q \cdot r) \ell_{2}^{\alpha \mu \nu}+\frac{p^{2}}{p^{2}+2(p \cdot q)} \ell_{3}^{\alpha \mu \nu}-(p \cdot r) \ell_{5}^{\alpha \mu \nu}+\frac{q^{2}}{q^{2}+2(q \cdot r)} \ell_{6}^{\alpha \mu \nu}-(p \cdot q) \ell_{8}^{\alpha \mu \nu}+\frac{r^{2}}{r^{2}+2(p \cdot r)} \ell_{9}^{\alpha \mu \nu}-\ell_{10}^{\alpha \mu \nu}, \\
& v_{\mathbf{T}}^{\alpha \mu \nu}=-\frac{2}{p^{2}+2(p \cdot q)} t_{1}^{\alpha \mu \nu}-\frac{2}{q^{2}+2(q \cdot r)} t_{2}^{\alpha \mu \nu}-\frac{2}{r^{2}+2(p \cdot r)} t_{3}^{\alpha \mu \nu} .
\end{aligned}
$$


[1] W. J. Marciano and H. Pagels, Phys. Rep. 36, 137 (1978).

[2] D. J. Gross and F. Wilczek, Phys. Rev. Lett. 30, 1343 (1973).

[3] H. D. Politzer, Phys. Rev. Lett. 30, 1346 (1973).

[4] J. M. Cornwall and J. Papavassiliou, Phys. Rev. D 40, 3474 (1989).

[5] R. Alkofer, C. S. Fischer, and F. J. Llanes-Estrada, Phys. Lett. B 611, 279 (2005).

[6] M. Binger and S. J. Brodsky, Phys. Rev. D 74, 054016 (2006).

[7] C. S. Fischer and J. M. Pawlowski, Phys. Rev. D 75, 025012 (2007).

[8] C. S. Fischer, J. Phys. G 32, R253 (2006).

[9] D. Binosi and J. Papavassiliou, J. High Energy Phys. 03 (2011) 121.

[10] M. Q. Huber and L. von Smekal, J. High Energy Phys. 04 (2013) 149.

[11] M. Q. Huber, A. Maas, and L. von Smekal, J. High Energy Phys. 11 (2012) 035.

[12] D. Binosi, D. Ibanez, and J. Papavassiliou, Phys. Rev. D 87, 125026 (2013).

[13] M. Pelaez, M. Tissier, and N. Wschebor, Phys. Rev. D 88, 125003 (2013).

[14] A. Blum, M. Q. Huber, M. Mitter, and L. von Smekal, Phys. Rev. D 89, 061703 (2014).

[15] G. Eichmann, R. Williams, R. Alkofer, and M. Vujinovic, Phys. Rev. D 89, 105014 (2014).

[16] R. Williams, C. S. Fischer, and W. Heupel, Phys. Rev. D 93, 034026 (2016).

[17] A. C. Aguilar, D. Ibañez, and J. Papavassiliou, Phys. Rev. D 87, 114020 (2013).

[18] A. C. Aguilar, D. Binosi, D. Ibañez, and J. Papavassiliou, Phys. Rev. D 89, 085008 (2014).

[19] A. L. Blum, R. Alkofer, M. Q. Huber, and A. Windisch, Acta Phys. Pol. B Proc. Suppl. 8, 321 (2015).

[20] A. L. Blum, R. Alkofer, M. Q. Huber, and A. Windisch, EPJ Web Conf. 137, 03001 (2017).

[21] A. K. Cyrol, L. Fister, M. Mitter, J. M. Pawlowski, and N. Strodthoff, Phys. Rev. D 94, 054005 (2016).

[22] L. Corell, A. K. Cyrol, M. Mitter, J. M. Pawlowski, and N. Strodthoff, SciPost Phys. 5, 066 (2018).

[23] A. Cucchieri, A. Maas, and T. Mendes, Phys. Rev. D 74, 014503 (2006).

[24] A. Cucchieri, A. Maas, and T. Mendes, Phys. Rev. D 77, 094510 (2008).

[25] A. Athenodorou, D. Binosi, P. Boucaud, F. De Soto, J. Papavassiliou, J. Rodriguez-Quintero, and S. Zafeiropoulos, Phys. Lett. B 761, 444 (2016).

[26] A. G. Duarte, O. Oliveira, and P. J. Silva, Phys. Rev. D 94, 074502 (2016).

[27] A. Sternbeck, P.-H. Balduf, A. Kizilersu, O. Oliveira, P. J. Silva, J.-I. Skullerud, and A. G. Williams, Proc. Sci., LATTICE2016 (2017) 349.

[28] P. Boucaud, F. De Soto, J. Rodriguez-Quintero, and S. Zafeiropoulos, Phys. Rev. D 95, 114503 (2017).

[29] M. Vujinovic and T. Mendes, Phys. Rev. D 99, 034501 (2019).

[30] J. M. Cornwall, Phys. Rev. D 26, 1453 (1982).

[31] A. C. Aguilar and A. A. Natale, J. High Energy Phys. 08 (2004) 057.
[32] A. C. Aguilar, A. A. Natale, and P. S. Rodrigues da Silva, Phys. Rev. Lett. 90, 152001 (2003).

[33] A. C. Aguilar, D. Binosi, and J. Papavassiliou, Phys. Rev. D 78, 025010 (2008).

[34] D. Binosi and J. Papavassiliou, Phys. Rep. 479, 1 (2009).

[35] J. M. Cornwall, J. Papavassiliou, and D. Binosi, The Pinch Technique and its Applications to Non-Abelian Gauge Theories (Cambridge University Press, Cambridge, England, 2010), Vol. 31.

[36] D. Binosi, D. Ibañez, and J. Papavassiliou, Phys. Rev. D 86, 085033 (2012).

[37] D. Ibañez and J. Papavassiliou, Phys. Rev. D 87, 034008 (2013).

[38] A. C. Aguilar, D. Binosi, and J. Papavassiliou, Front. Phys. 11, 111203 (2016).

[39] A. C. Aguilar, D. Binosi, C. T. Figueiredo, and J. Papavassiliou, Phys. Rev. D 94, 045002 (2016).

[40] D. Binosi and J. Papavassiliou, Phys. Rev. D 97, 054029 (2018).

[41] A. C. Aguilar, D. Binosi, C. T. Figueiredo, and J. Papavassiliou, Eur. Phys. J. C 78, 181 (2018).

[42] F. Gao, S.-X. Qin, C. D. Roberts, and J. RodriguezQuintero, Phys. Rev. D 97, 034010 (2018).

[43] J. Meyers and E. S. Swanson, Phys. Rev. D 87, 036009 (2013).

[44] S. Strauss, C. S. Fischer, and C. Kellermann, Phys. Rev. Lett. 109, 252001 (2012).

[45] H. Sanchis-Alepuz, C. S. Fischer, C. Kellermann, and L. von Smekal, Phys. Rev. D 92, 034001 (2015).

[46] H. Fukamachi, K.-I. Kondo, S. Nishino, and T. Shinohara, Prog. Theor. Exp. Phys. 2017, 053B05 (2017).

[47] S.-S. Xu, Z.-F. Cui, L. Chang, J. Papavassiliou, C. D. Roberts, and H.-S. Zong, arXiv:1805.06430.

[48] M. Vujinovic, R. Alkofer, G. Eichmann, and R. Williams, Acta Phys. Pol. B Proc. Suppl. 7, 607 (2014).

[49] C. D. Roberts and A. G. Williams, Prog. Part. Nucl. Phys. 33, 477 (1994).

[50] R. Alkofer and L. von Smekal, Phys. Rep. 353, 281 (2001).

[51] I. C. Cloet and C. D. Roberts, Prog. Part. Nucl. Phys. 77, 1 (2014).

[52] D. Binosi, L. Chang, J. Papavassiliou, and C. D. Roberts, Phys. Lett. B 742, 183 (2015).

[53] C. J. Morningstar and M. J. Peardon, Phys. Rev. D 60, 034509 (1999).

[54] J. S. Ball and T.-W. Chiu, Phys. Rev. D 22, 2550 (1980).

[55] W. Schleifenbaum, A. Maas, J. Wambach, and R. Alkofer, Phys. Rev. D 72, 014017 (2005).

[56] D. Binosi, L. Chang, J. Papavassiliou, S.-X. Qin, and C. D. Roberts, Phys. Rev. D 95, 031501 (2017).

[57] F. T. Hawes, P. Maris, and C. D. Roberts, Phys. Lett. B 440, 353 (1998).

[58] L. Chang and C. D. Roberts, Phys. Rev. Lett. 103, 081601 (2009).

[59] S.-x. Qin, L. Chang, Y.-x. Liu, C. D. Roberts, and D. J. Wilson, Phys. Rev. C 84, 042202 (2011).

[60] A. K. Cyrol, M. Mitter, J. M. Pawlowski, and N. Strodthoff, Phys. Rev. D 97, 054006 (2018).

[61] A. Salam, Phys. Rev. 130, 1287 (1963).

[62] A. Salam and R. Delbourgo, Phys. Rev. 135, B1398 (1964). 
[63] R. Delbourgo and P. C. West, J. Phys. A 10, 1049 (1977).

[64] R. Delbourgo and P. C. West, Phys. Lett. 72B, 96 (1977).

[65] G. Eichmann and G. Ramalho, Phys. Rev. D 98, 093007 (2018).

[66] J. Skullerud and A. Kizilersu, J. High Energy Phys. 09 (2002) 013.

[67] A. Cucchieri and T. Mendes, Proc. Sci., LAT2007 (2007) 297.

[68] A. Cucchieri and T. Mendes, Proc. Sci., QCD-TNT09 (2009) 026.

[69] A. Cucchieri and T. Mendes, Phys. Rev. Lett. 100, 241601 (2008).

[70] I. L. Bogolubsky, E. M. Ilgenfritz, M. Muller-Preussker, and A. Sternbeck, Proc. Sci., LATTICE2007 (2007) 290.

[71] I. Bogolubsky, E. Ilgenfritz, M. Muller-Preussker, and A. Sternbeck, Phys. Lett. B 676, 69 (2009).

[72] O. Oliveira and P. Silva, Proc. Sci., LAT2009 (2009) 226.

[73] O. Oliveira, P. J. Silva, J.-I. Skullerud, and A. Sternbeck, arXiv: 1809.02541.

[74] O. Oliveira and P. Bicudo, J. Phys. G 38, 045003 (2011).

[75] O. Oliveira and P. J. Silva, Phys. Rev. D 86, 114513 (2012).

[76] P. O. Bowman, U. M. Heller, D. B. Leinweber, M. B. Parappilly, A. G. Williams, and J.-b. Zhang, Phys. Rev. D 71, 054507 (2005).

[77] A. Ayala, A. Bashir, D. Binosi, M. Cristoforetti, and J. Rodriguez-Quintero, Phys. Rev. D 86, 074512 (2012).

[78] P. Boucaud, J. P Leroy, A. Le Yaouanc, J. Micheli, O. Pène, and J. Rodríguez-Quintero, J. High Energy Phys. 06 (2008) 099.

[79] D. Dudal, J. A. Gracey, S. P. Sorella, N. Vandersickel, and H. Verschelde, Phys. Rev. D 78, 065047 (2008).

[80] C. S. Fischer, A. Maas, and J. M. Pawlowski, Ann. Phys. (Amsterdam) 324, 2408 (2009).

[81] M. Mitter, J. M. Pawlowski, and N. Strodthoff, Phys. Rev. D 91, 054035 (2015).

[82] F. Siringo, Nucl. Phys. B907, 572 (2016).

[83] M. Q. Huber, arXiv:1808.05227.

[84] A. C. Aguilar, M. N. Ferreira, C. T. Figueiredo, and J. Papavassiliou, Phys. Rev. D 99, 034026 (2019).

[85] A. C. Aguilar, D. Binosi, and J. Papavassiliou, Phys. Rev. D 84, 085026 (2011).

[86] A. C. Aguilar, D. Binosi, and J. Papavassiliou, Phys. Rev. D 89, 085032 (2014).

[87] A. C. Aguilar, D. Ibanez, V. Mathieu, and J. Papavassiliou, Phys. Rev. D 85, 014018 (2012).

[88] A. I. Davydychev, P. Osland, and O. V. Tarasov, Phys. Rev. D 54, 4087 (1996).

[89] K.-I. Kondo, Phys. Lett. B 514, 335 (2001).

[90] A. C. Aguilar, D. Binosi, and J. Papavassiliou, Phys. Rev. D 95, 034017 (2017).

[91] F. Siringo and G. Comitini, Phys. Rev. D 98, 034023 (2018).

[92] B. W. Mintz, L. F. Palhares, G. Peruzzo, and S. P. Sorella, Phys. Rev. D 99, 034002 (2019).

[93] A. Cucchieri, T. Mendes, and E. M. Santos, Phys. Rev. Lett. 103, 141602 (2009).

[94] P. Bicudo, D. Binosi, N. Cardoso, O. Oliveira, and P. J. Silva, Phys. Rev. D 92, 114514 (2015).
[95] A. Cucchieri, D. Dudal, T. Mendes, O. Oliveira, M. Roelfs, and P. J. Silva, arXiv:1812.00429.

[96] A. Pilaftsis, Nucl. Phys. B487, 467 (1997).

[97] D. Binosi and J. Papavassiliou, Phys. Rev. D 66, 111901 (2002).

[98] D. Binosi and J. Papavassiliou, J. Phys. G 30, 203 (2004).

[99] B. S. DeWitt, Phys. Rev. 162, 1195 (1967).

[100] J. Honerkamp, Nucl. Phys. B48, 269 (1972).

[101] R. E. Kallosh, Nucl. Phys. B78, 293 (1974).

[102] H. Kluberg-Stern and J. B. Zuber, Phys. Rev. D 12, 482 (1975).

[103] I. Y. Arefeva, L. D. Faddeev, and A. A. Slavnov, Theor. Math. Phys. 21, 1165 (1974).

[104] G. 't Hooft, in Karpacz 1975, Proceedings, Acta Universitatis Wratislaviensis No. 368, Vol. 1, Wroclaw 1976 (Wroclaw University, Wroclaw, 1976), pp. 345-369.

[105] L. F. Abbott, Nucl. Phys. B185, 189 (1981).

[106] L. F. Abbott, Acta Phys. Pol. B 13, 33 (1982).

[107] J. S. Schwinger, Phys. Rev. 125, 397 (1962).

[108] J. S. Schwinger, Phys. Rev. 128, 2425 (1962).

[109] R. Jackiw and K. Johnson, Phys. Rev. D 8, 2386 (1973).

[110] R. Jackiw, in Proceedings, Laws Of Hadronic Matter, Erice, 1975 (MIT, Cambridge, MA, 1973), p. 23.

[111] J. M. Cornwall and R. E. Norton, Phys. Rev. D 8, 3338 (1973).

[112] E. Eichten and F. Feinberg, Phys. Rev. D 10, 3254 (1974).

[113] E. C. Poggio, E. Tomboulis, and S. H. H. Tye, Phys. Rev. D 11, 2839 (1975).

[114] J. Smit, Phys. Rev. D 10, 2473 (1974).

[115] A. C. Aguilar and J. Papavassiliou, Phys. Rev. D 81, 034003 (2010).

[116] P. A. Grassi, T. Hurth, and M. Steinhauser, Ann. Phys. (N.Y.) 288, 197 (2001).

[117] D. Binosi, C. Mezrag, J. Papavassiliou, C. D. Roberts, and J. Rodriguez-Quintero, Phys. Rev. D 96, 054026 (2017).

[118] P. Boucaud, F. De Soto, J. Leroy, A. Le Yaouanc, J. Micheli, O. Pène, and J. Rodríguez-Quintero, Phys. Rev. D 79, 014508 (2009).

[119] J. C. Taylor, Nucl. Phys. B33, 436 (1971).

[120] A. C. Aguilar, J. C. Cardona, M. N. Ferreira, and J. Papavassiliou, Phys. Rev. D 98, 014002 (2018).

[121] C. de Boor, A Practical Guide to Splines, Applied Mathematical Sciences (Springer, New York, 2001).

[122] W. Celmaster and R. J. Gonsalves, Phys. Rev. D 20, 1420 (1979).

[123] Y. Takahashi, in Proceedings, International Symposium on Quantum Field Theory, Positano, Italy (Amsterdam, North-Holland, 1986), p. 019.

[124] K.-I. Kondo, Int. J. Mod. Phys. A 12, 5651 (1997).

[125] H.-X. He and F. Khanna, Int. J. Mod. Phys. A 21, 2541 (2006).

[126] S.-X. Qin, L. Chang, Y.-X. Liu, C. D. Roberts, and S. M. Schmidt, Phys. Lett. B 722, 384 (2013).

[127] A. C. Aguilar, D. Binosi, D. Ibañez, and J. Papavassiliou, Phys. Rev. D 90, 065027 (2014).

[128] L. Albino, A. Bashir, L. X. G. Guerrero, B. E. Bennich, and E. Rojas, arXiv:1812.02280.

[129] J. A. Gracey, Phys. Rev. D 84, 085011 (2011). 